\title{
Serum Exosomal microRNA Profiling in AIDS Complicated with Talaromyces marneffei Infection
}

\author{
Qiu- Yue Ning ${ }^{1, *}$ \\ $\mathrm{Na} \mathrm{Liu}{ }^{2}, *$ \\ Ji-Zhou Wu' \\ Die-Fei Hu' \\ Qi Wei ${ }^{1}$ \\ Jin-Ai Zhou ${ }^{3}$ \\ Jun Zou ${ }^{4}$ \\ Ning Zang ${ }^{5,6}$ \\ Guo-Jian Li' \\ 'Department of Infectious Diseases, The \\ First Affiliated Hospital of Guangxi \\ Medical University, Nanning, Guangxi, \\ People's Republic of China; ${ }^{2}$ Life Sciences \\ Institute, Guangxi Medical University, \\ Nanning, Guangxi, People's Republic of \\ China; ${ }^{3}$ Youjiang Medical University for \\ Nationalities, Baise, Guangxi, People's \\ Republic of China; ${ }^{4}$ The Fourth People's \\ Hospital of Nanning, Nanning, Guangxi, \\ People's Republic of China; ${ }^{5}$ School of \\ Basic Medicine, Guangxi Medical \\ University, Nanning, Guangxi, People's \\ Republic of China; 'Key Laboratory of \\ AIDS Prevention and Treatment, Guangxi \\ Medical University, Nanning, Guangxi, \\ People's Republic of China
}

*These authors contributed equally to this work
Correspondence: Guo-Jian Li; Ning Zang Tel +86 77l-535653I

Email liguojian2016@yeah.net;

zangning@gxmu.edu.cn
Introduction: In order to find the early diagnostic markers of AIDS combined with TM infection, we detected and analyzed the serum exosomal miRNAs of AIDS patients with or without TM infection.

Materials and Methods: We profiled the expression levels of miRNAs via RNA sequencing in serum exosomes from the pooled samples of 17 AIDS patients combined without TM infection and 15 AIDS combined with TM infection patients. For external validation, we validated these results using real-time quantification polymerase chain reaction (qRT-PCR) in an independent cohort of 35 AIDS patients combined without TM infection and 33 AIDS combined with TM infection patients. Finally, bioinformatics was used to predict the target genes and pathways of meaningful miRNAs.

Results: A total of 131 serum exosomal miRNAs including 73 up-regulated and 59 downregulated miRNAs were found to be differentially expressed $(\log 2 F C \geq 1$ and FDR $<0.01)$ in the two groups. A validation analysis revealed that three miRNAs (miR-192-5p, miR-194-5p and miR-1246) were upregulated in exosomes from AIDS combined with TM infection patients. ROC analyses showed that the AUC in combined diagnosis of the three miRNAs was 0.742 , and the diagnostic sensitivity and specificity were 0.568 and 0.861 , respectively. In the biological process analysis, all the 3 miRNAs were involved in transcription, DNA-templated and positive regulation of transcription from RNA polymerase II promoter. At the same time, the related pathways were involved in TGF- $\beta$ signaling pathway, AMPK signaling pathway, Wnt signaling pathway, MAPK signaling pathway, cGMP-PKG and cAMP signaling pathway, etc.

Conclusion: miR-192-5p, miR-194-5p and miR-1246 in serum exosomes might be a potential biomarker for AIDS combined with TM infection patients, which may be involved in TGF- $\beta$ signaling pathway, AMPK signaling pathway, Wnt signaling pathway, MAPK signaling pathway, cGMP-PKG and cAMP signaling pathway, etc. Further research is needed on the biological functions and mechanisms of these miRNAs.

Keywords: exosomes, AIDS, Talaromyces marneffei, microRNA, biomarkers

\section{Introduction}

The prevalence of Human immunodeficiency virus/Acquired Immune Deficiency Syndrome (HIV/AIDS) is still a public health problem that threatens the health of all human beings. According to the latest report of the World Health Organization, in the end of 2019, there were 38 million patients with human immunodeficiency virus/acquired immunodeficiency syndrome (HIV/AIDS) all over the world. ${ }^{1}$ In 2019, there were about 71,204 new HIV/AIDS patients and about 20,999 people died from AIDS in China, ${ }^{2}$ while there were more than 30,000 new HIV-1 infections every year in Guangxi ${ }^{3}$ which was one of the highrisk areas. ${ }^{4}$ 
After HIV-1 infection, it mainly attacks the cellular immune system of the human body, leading to a decline in $\mathrm{CD}^{+} \mathrm{T}$ lymphocytes, which causes immune function defects. When HIV-1 patients progress to the AIDS stage, they are prone to various opportunistic infections, and Talaromyces marneffei (TM) is one of the common fungal infections. TM infections in China had obvious local epidemic characteristics and were mainly concentrated in the south, such as Guangxi, Guangdong, Hong Kong and Taiwan, ${ }^{5}$ among which Guangxi had an incidence of $42.8 \%{ }^{6}$ In addition, it was reported that among HIV-1 positive patients in Guangxi, the infection rate of TM would be greater than $30 \%$ when the count of $\mathrm{CD} 4^{+} \mathrm{T}$ lymphocytes was less than $50 / \mu \mathrm{L} .^{7}$ For patients with AIDS combined with TM infection, early diagnosis and timely antifungal combined ART treatment can effectively control the disease progression. However, the mortality rate of those who had not been diagnosed and treated in time was very high. According to statistics, the mortality rate of TM patients after receiving antifungal treatment was $24.3 \%$ while the mortality rate of patients who did not receive antifungal treatment was reached to $50.6 \%,{ }^{6}$ and there were reports that the mortality rate of patients who had not received timely treatment even reached to $91.3 \%{ }^{8}$ At present, the diagnosis of TM infection mainly relied on pathogenic microscopy and culture, but the microscopic detection rate was low and the fungal culture took a long time, so early diagnosis of TM infection was very difficult.

Exosomes are nanovesicles released from different cell types, with a diameter of about $30-150 \mathrm{~nm}$, which are present in various body fluids including blood, urine, saliva, tears, semen, breast milk, and ascites, etc. Exosomes contain a variety of molecules such as proteins, lipids, and nucleic acids, etc. that exhibit biological activity and reflect the state and type of their original cells. Fungi, bacteria, viruses and other microorganisms can not only secrete exosomes that are used to spread the infection and avoid the host's immune system, but also stimulate the production of exosomes in host cells. ${ }^{9,10}$ Therefore, as a product of the interaction between host immune cells and pathogens, exosomes simultaneously carry information from the host and pathogens, thus becoming a research hotspot. The content of exosomes changes with diseases or treatments. They act as signal organelles between cells, participate in many physiological and pathological processes, and have potential roles in clinical diagnosis and treatment. ${ }^{11}$
As one of nucleic acid cargo of exosomes, microRNA (miRNA), which was first discovered in Caenorhabditis elegans, ${ }^{12}$ is a type of endogenous short-stranded noncoding RNA with regulatory functions, containing 18-25 nucleotides and can affect the degradation of mRNA complementary sequences and regulate gene expression after transcription. miRNA plays an important immunomodulatory role in TM infection ${ }^{13-15}$ and the transfer of miRNA mediated by exosomes is also regarded as a genetic communication mechanism between cells. ${ }^{16,17}$ Besides, the secretion and uptake of miRNA is not a random process but a controlled process, so it can provide new research directions for the pathogenesis of HIV-1, disease progression, related inflammation, the efficacy of ART, and therapies aimed at reducing immune activation, and so on. ${ }^{18}$ Therefore, this experiment intends to explore the serum exosomes of patients with AIDS and TM infection, in order to find potential biomarkers for rapid diagnosis of TM infection, and to provide new diagnostic methods for early clinical diagnosis and early treatment of TM patients.

\section{Materials and Methods Ethics Statement}

The study was reviewed and approved by the First Affiliated Hospital of Guangxi Medical University Ethical Review Committee. All research subjects signed the relevant informed consent in written form before being enrolled, and clinical investigations were conducted according to the principles of the Helsinki Declaration.

\section{Human Subject}

The research subjects were divided into an experimental group and control group. All selected subjects were HIVpositive patients from the First Affiliated Hospital of Guangxi Medical University and the Fourth People's Hospital, and the $\mathrm{CD}^{+} 4 \mathrm{~T}$ cell count was less than or equal to 200 cells $/ \mu \mathrm{L}$. The diagnosis of all AIDS patients meets the diagnostic criteria of the Chinese AIDS Diagnosis and Treatment Guidelines (2018 Edition). Laboratory testing of hepatitis B surface antigen (HBsAg), hepatitis B core antibody ( $\mathrm{HBc}-\mathrm{Ab}$ ), hepatitis $\mathrm{C}$ antibody (HCV-Ab), cytomegalovirus (Cytomegalovirus, CMV) DNA, Treponema pallidum particle agglutination (TPPA) test, Epstein-Barr virus antibody (EBV-Ab) and EBV-RNA were all negative. Patients with Talaromyces marneffei (TM) cultured in clinical specimens were included in the experimental group, and patients with T.M cultured were included in the control 
group. All patients were not treated with antiretroviral therapy, and excluded malignant tumors and other deep fungal infections such as Cryptococcus, Candida, Aspergillus, Pneumocystis and Mucor, etc. Finally, the test cohort included 15 AIDS combined with TM infection patients in the experimental group and 17 AIDS combined without TM infection patients in the control group while the validation cohort included 33 AIDS combined with TM infection patients in the experimental group and 35 AIDS combined without TM infection patients in the control group. The validation cohort included all subjects in the test cohort.

\section{Serum Collection and Separation}

The researchers' blood samples were collected in biochemical tubes containing coagulants, transported on ice, centrifuged at $4^{\circ} \mathrm{C}$ and $3000 \mathrm{rpm}$ for 15 minutes, and serum was collected and stored at $-80^{\circ} \mathrm{C}$ until further use. All blood samples were processed within 12 hours after collection.

\section{Exosome Isolation from Serum}

Exosomes were isolated from serum using the ExoQuick exosome precipitation solution as per manufacturer's recommendations (SBI, USA). ${ }^{19}$ Frozen serum samples were taken out from $-80^{\circ} \mathrm{C}$ fridge, thawed at $25^{\circ} \mathrm{C}$, and centrifuged at $4^{\circ} \mathrm{C}, 3000 \mathrm{rpm}$ for 15 minutes to remove cell debris. Then, about $500 \mu \mathrm{L}$ volume of the supernatant was transferred to a new tube, and $125 \mu \mathrm{L}$ volume of ExoQuick exosome precipitation solution was added, vortex-mixed and incubated for 30 minutes at $4^{\circ} \mathrm{C}$. After incubation, the samples were centrifuged at $4^{\circ} \mathrm{C}, 13000 \mathrm{rpm}$ for 2 minutes. Without disrupting the pellet, the supernatant was removed and discarded. The exosomes in the pellets were dissolved in $200 \mu \mathrm{L}$ volume of PBS and stored at $-80^{\circ} \mathrm{C}$.

\section{Transmission Electron Microscopy (TEM)}

At room temperature, $20 \mu \mathrm{L}$ volumes of exosomes were dropped on a copper mesh with a diameter of $2 \mathrm{~nm}$ for 10 min, dried with filter paper, and stained with $3 \%$ phosphotungstic acid solution for $2 \mathrm{~min}$ and air-dried. After the copper mesh was installed in the sample slot of the transmission electron microscope, the focus and brightness were adjusted to observe and photograph the sample, and the vesicle structure and the shape of exosomes were analyzed. All TEM experiments were performed at the Facilities of the Transmission Electron Microscopy Laboratory of Guangxi Medical University.

\section{Dynamic Light Scattering (DLS)}

Size distribution and the polydispersity index of isolated serum exosomes were detected using a Zetasizer Nano ZS system (Malvern Instruments, Malvern, U.K.). ${ }^{20}$ In Zetasizer Software, after setting the parameters, the measuring cell was cleaned with ultrapure water, the sample was filtered with a $0.22 \mu \mathrm{m}$ syringe filter, and $40 \mu \mathrm{L}$ exosomes were placed in the measuring cell for measurement.

\section{Western Blotting}

Exosomes isolated from serum samples were lysed with Exosomes dedicated lysate (Umibio, China) at $4^{\circ} \mathrm{C}$ and the protein content was determined using the BCA protein concentration determination kit (Solarbio, China). SDS Loading Buffer (5X) was added to the samples and incubated at $95^{\circ} \mathrm{C}$ for $6 \mathrm{~min}$. Sixty micrograms of exosomal protein per well was separated by sodium dodecyl sulfatepolyacrylamide gel electrophoresis (SDS-PAGE) and transferred onto polyvinylidene fluoride membranes (PVDF). Then, PVDF membranes were washed with $1 \times$ TBST buffer $(\mathrm{pH}$ 7.6) and blocked with $4 \%$ milk at room temperature for $1 \mathrm{~h}$. After PVDF membranes were washed with TBST buffer again, PVDF membranes were incubated with the primary antibody CD9, CD63 and calnexin (Abcam, USA) overnight at $4^{\circ} \mathrm{C}$. Finally, PVDF membranes were incubated with fluorescent secondary antibody (Shanghai Zhennuo Biological Company, China) for $2 \mathrm{~h}$ at room temperature, and visualized with the Odyssey dual-color infrared fluorescence imaging system (LI-COR Company, USA).

\section{Exosome RNA Isolation}

Exosomal RNA was isolated with the total exosome RNA isolation kit (System Biosciences, CA, USA) as per the manufacturer's instructions. ${ }^{19}$ Total RNA concentration was measured using the NanoDrop 2000 spectrophotometer (Thermo Scientific, MA, USA).

\section{RNA Sequencing}

According to the recommendations of the manufacturer, barcoded small RNA-Seq libraries were prepared using the Truseq $^{\mathrm{TM}}$ Small RNA sample prep Kit (Illumina, CA) from $1 \mu \mathrm{g}$ serum exosome RNA and recovered by $6 \%$ Novex TBE PAGE gel, $1.0 \mathrm{~mm}, 10$ well (Invitrogen, $\mathrm{CA})$. The recovered libraries were quantified through TBS380 (Picogreen), and putted on the machine after 
mixing according to the data ratio. Thereafter, bridge PCR amplification was performed on cBot to generate clusters, and SE50 sequencing was performed on the HiSeq sequencing platform. The entire sequencing process was implemented by Major Biotech, Shanghai, China.

\section{qRT-PCR Validation of miRNAs}

To validate candidate miRNAs from sequencing data, we performed qRT-PCR analysis of miR-28-3p, miR-144-3p, miR-192-5p, miR-194-5p, miR-199a-3p, miR-455-3p, miR-486-5p and miR-1246, while U6 was used as the internal control. The miRNA-specific primers were all designed by Nanning Kedi Company of Guangxi, China, and synthesized by TAKARA company. The specific primer of U6 was provided by TAKARA. Equal volumes of exosomal RNA $(10 \mu \mathrm{L})$ from each sample were reverse transcribed using the $\operatorname{Mir}-\mathrm{X}^{\mathrm{TM}}$ miRNA First Strand Synthesis Kit (TAKARA) at $37^{\circ} \mathrm{C}$ for 60 min, and then the enzyme was inactivated at $85^{\circ} \mathrm{C}$ for 5 min. After denatured template DNA at $95^{\circ} \mathrm{C}$ for $10 \mathrm{~s}, 40$ cycles of $95^{\circ} \mathrm{C}$ for $5 \mathrm{~s}$, and $60^{\circ} \mathrm{C}$ for $20 \mathrm{~s}$ were performed using TB Green ${ }^{\circledR}$ Premix Ex TaqTM II Kit (TAKARA), ${ }^{21}$ on the ABI StepOne/StepOnePlus Real-Time System. Ct values were normalized $(\Delta \mathrm{Ct})$ by the global mean and $2^{-\Delta \Delta \mathrm{Ct}}$ calculated to account for the difference between groups.

\section{Statistical Analysis}

Continuous variables were expressed in mean \pm standard deviation. Group means of clinical and qRT-PCR miRNA measurement data were compared using independent sample $t$-test, and the $\mathrm{X}^{2}$ test was used for the count data. If the

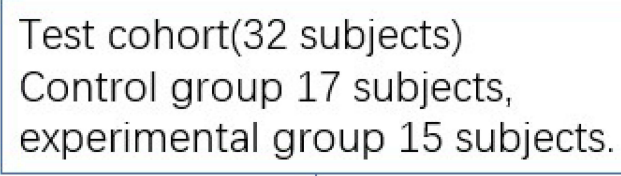

Validation cohort(68 subjects, including the subjects of test cohort) Control group 35 subjects, experimental group 33 subjects.

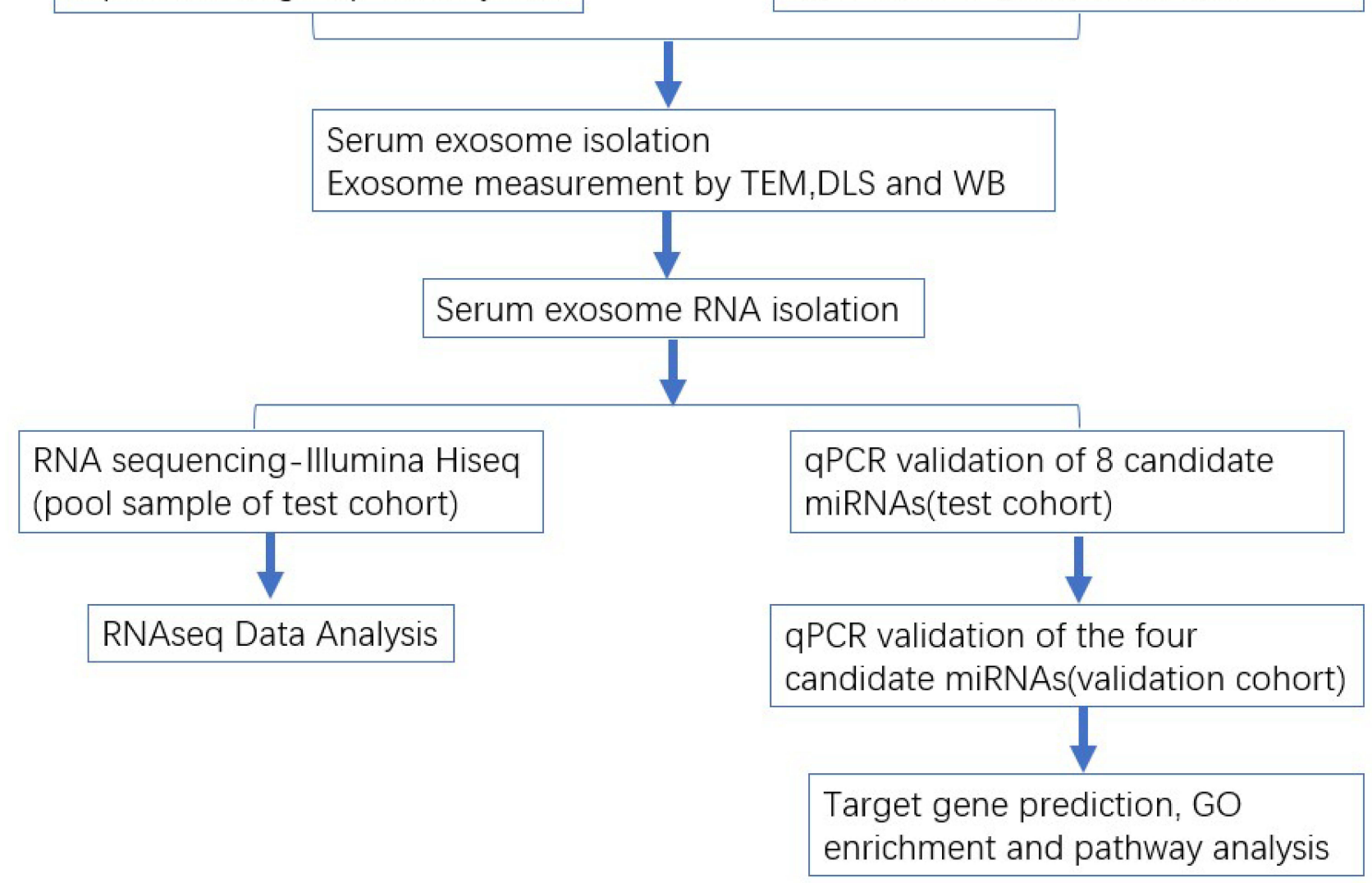

Figure I Study design summarizing the cohort, methodology, and data analysis. $500 \mu \mathrm{L}$ serum from subjects in test or validation cohorts were used to isolate exosomes using the ExoQuick kit. Exosomes were measured by TEM and WB. Small RNA libraries were prepared and sequenced on HiSeq, and candidate miRNAs were validated by qRT-PCR. 
data were non-normally distributed, we used Wilcoxon test. Evaluation individually or the logistics binary regression model were used to integrate variables, and at the same time receiver operator characteristic curve (ROC) was drawn. All data analyses and graphical representations were performed and generated in GraphPad Prism 5.0 and Software SPSS 20.0. Exosomal miRNA ratio analysis between the two groups was selected based on an arbitrary $|\log 2 \mathrm{FC}| \geq 1$ and False Discovery Rate (FDR) $<0.01$ with all expression data represented in $\log 2$ scale using the DESeq analysis software, and verification of candidate miRNAs was performed on qRT-PCR while bioinformatics analysis was analyzed on imageGP online software. In addition, TargetScanHuman7.2 and starBase V2.0 database were used to predict target genes, and the online gene function annotation tool on the DAVID website was used to perform GO enrichment for differential genes while the related signal transduction pathways were analyzed through KEGG database (interactions with a p-value $<0.05$ were considered).

\section{Results}

\section{Clinical Characteristics of Participants}

A total of 17 and 15 subjects comprised the control and experimental group in the test cohort, respectively, while a total of 35 and 33 subjects comprised the control and experimental group in the verification cohort (including the subjects of the test cohort), respectively (Figure 1). A comparative analysis of all the clinically collected data showed that there was no significant difference in the number of age, sex, white blood cells, neutrophils, lymphocytes, and platelets between the control group and the experimental group, while the monocyte count, $\mathrm{CD}^{+} \mathrm{T}$ lymphocyte count, $\mathrm{CD}^{+} \mathrm{T}$ lymphocyte count and CD4/CD8 ratio were significant differences between the control group and the experimental group. The number of monocyte count, $\mathrm{CD}^{+} \mathrm{T}$ lymphocyte count, $\mathrm{CD}^{+} \mathrm{T}$ lymphocyte count and $\mathrm{CD} 4 / \mathrm{CD} 8$ ratio in the experimental group was significantly lower than the control group (Table 1).

\section{Characterization of Serum Exosomes}

Exosomes were isolated from subjects' serum using the ExoQuick exosome precipitation solution and characterized by transmission electron microscopy (TEM) and Western blotting for exosome markers (Figure 2). TEM revealed vesicles of $50-150 \mathrm{~nm}$ in diameter (Figure 2A),
Table I Clinical Characteristics of Participants in Validation Cohort

\begin{tabular}{|c|c|c|c|}
\hline Variables & Control Group & $\begin{array}{l}\text { Experimental } \\
\text { Group }\end{array}$ & $P$ or $X^{2}$ \\
\hline Diagnosis time & $<$ month & $<1$ month & - \\
\hline Disease stage & AIDS period & AIDS period & - \\
\hline CD4 count (cells/ $\mu \mathrm{L})$ & $167.83 \pm 103.36$ & $19.61 \pm 18.82$ & 0.00 \\
\hline CD8 count (cells/ $\mu \mathrm{L})$ & $828.26 \pm 552.71$ & $247.91 \pm 186.53$ & 0.00 \\
\hline CD4/CD8 ratio & $0.24 \pm 0.18$ & $0.073 \pm 0.052$ & 0.00 \\
\hline Blood cells $\left(10^{9} / \mathrm{L}\right)$ & $4.92 \pm 2.02$ & $4.01 \pm 2.46$ & 0.123 \\
\hline Neutrophils $\left(10^{9} / \mathrm{L}\right)$ & $2.44 \pm 1.72$ & $2.72 \pm 2.33$ & 0.577 \\
\hline Monocyte $\left(10^{9} / \mathrm{L}\right)$ & $1.30 \pm 1.18$ & $0.55 \pm 0.40$ & 0.002 \\
\hline Lymphocytes $\left(10^{9} / \mathrm{L}\right)$ & $0.57 \pm 0.36$ & $0.44 \pm 0.36$ & 0.167 \\
\hline Platelets $\left(10^{9} / \mathrm{L}\right)$ & $217.35 \pm 96.85$ & $170.78 \pm 101.60$ & 0.077 \\
\hline Age (years) & $48.73 \pm 15.48$ & $42.70 \pm|4.1|$ & 0.103 \\
\hline Sex (Female/male) & $12 / 23$ & $12 / 2 \mid$ & 0.858 \\
\hline
\end{tabular}

Notes: The measurement data was expressed as mean \pm standard deviation, and was tested by $t$-test. Counting data were expressed as numbers and tested by the chi-square test. Bold in the table indicates important data.

consistent with the size range of exosomes. The DLS chart showed that the main peak was in the range of about $80 \mathrm{~nm}$, and the density of impurity peaks was low, which was in line with the size of the exosomes, and indicated that the purity was acceptable (Figure 2B). In the Western blotting detection, serum exosomal markers CD9 and CD63 can be clearly displayed while calnexin was absent in the two groups (Figure 2C).

\section{Small RNA Classes and Distribution in Serum Exosomes of the Experimental Group and Control Group Patients}

In order to verify the serum exosomal miRNA expression profile of AIDS patients combined with T.M infection, 2 small RNA libraries of mixed samples in each group (17 control samples and 15 experimental samples) were constructed for deep sequencing. After removal of poor-quality raw reads, total clean reads were obtained for subsequent bioinformatics analyses. Table 2 shows that the sequence reads were produced from all samples, with the single base sequencing error rate below 0.1\%. Both Q20 and Q30 of each sample were $>80 \%$. Size distribution, base preference and distribution among mapped reads in each sample were analyzed further. Figures $3 \mathrm{~A}$ and B showed that most length was between $18 \mathrm{nt}$ and $32 \mathrm{nt}$ in the two samples, most of which were concentrated between $18 \mathrm{nt}$ and $24 \mathrm{nt}$, corresponding to miRNA and siRNA. No significant deviations in $\mathrm{GC}$ were found in Figures $3 \mathrm{C}$ and D. To examine the diversity of serum exosomal RNA cargo, reads were 

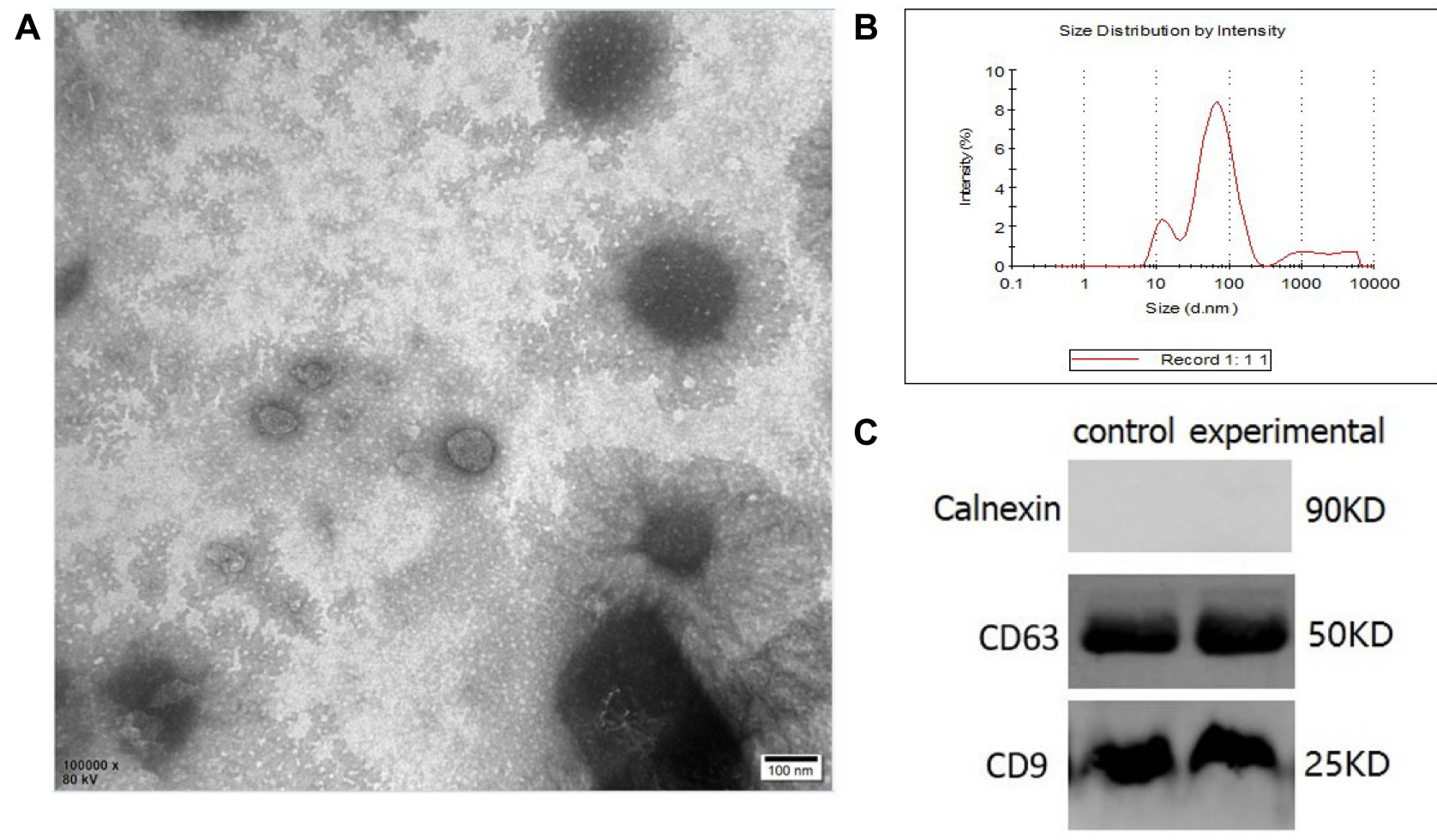

Figure 2 Characterization of exosome isolated from serum. (A) TEM image of isolated exosome from human serum. (B) DLS image of isolated exosome from human serum. (C) Detection of antibody CD9, CD63 and calnexin by Western blotting in pooled control $(n=17)$ and experimental $(n=15)$ samples.

mapped to the Rfam database for miRNA, rRNA, tRNA, snRNA, etc. Figures $3 \mathrm{E}$ and $\mathrm{F}$ showed the distributions of different RNA species identified in serum exosomes of control group and experimental group, respectively.

\section{Analysis of miRNA Profiles in Serum Exosomes}

Excluding non-miRNAs, the expression of identified miRNAs was standardized using TPM (transcripts per million; miRNAs were normalized by TPM). The miRNAs of the two groups were ranked according to the

Table 2 Statistical results of Sequencing Original Data

\begin{tabular}{|l|c|c|}
\hline Group & Control Group & Experimental Group \\
\hline Total Reads & $12,080,572$ & $12,317,250$ \\
Total Bases (bp) & $616,109,172$ & $628,179,750$ \\
Error\% & 0.0422 & 0.0438 \\
Q20\% & 91.38 & 90.86 \\
Q30\% & 82.92 & 82.23 \\
GC\% & 38.17 & 38.63 \\
\hline
\end{tabular}

Abbreviations: Q20, Q30\%, the percentage of bases with Phred value greater than $20 / 30$ in the total base; GC\%, the percentage of $G$ and $C$ base number in the total number of bases. expression level. Among the top ten miRNAs, miR-126-5p was the most abundant miRNA, followed by miR-486-5p and let-7a-5p in the control group (Figure 4A) while miR$122-5 p$ was the most abundant miRNA, followed by let$7 \mathrm{a}-5 \mathrm{p}$ and miR-126-5p in experimental group (Figure 4B). The types and abundance of miRNAs expressed in different tissues were different. Consequently, miRNAs were screened according to certain criteria (TMP $\geq 20$ ) to identify miRNAs that were specifically and co-expressed in each group of samples. The results showed that 245 known miRNAs were common between control and experimental group, and 58 and 63 were unique to control and experimental groups (Figure 4C).

\section{Differentially Expressed miRNAs}

Next, we investigated the miRNAs associated with AIDS combined with T.M infection. Cluster analysis and principal component analysis (PCA) were performed on the miRNAs of the two groups. The results showed that the miRNAs of the experimental group and the control group were clustered on both sides (Figure 5A), and the principal components of the two groups were quite different (Figure 5B). Further differential expression analysis for screening using DEGseq 


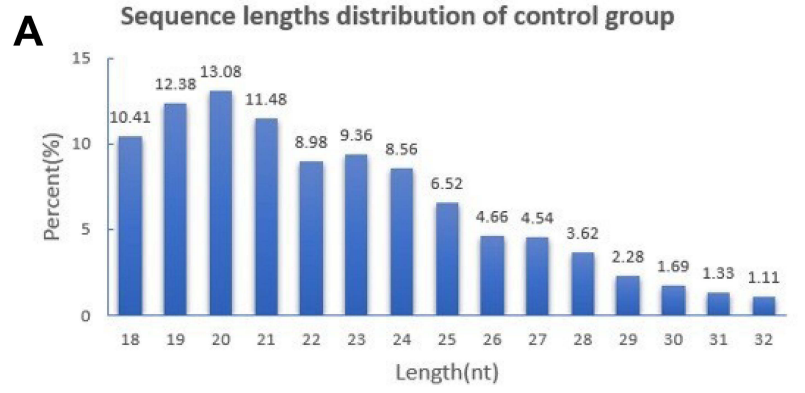

C

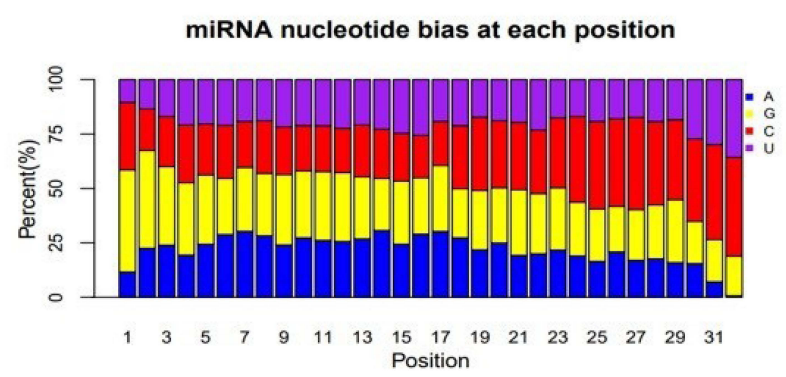

E

Mapped RNAs of control group

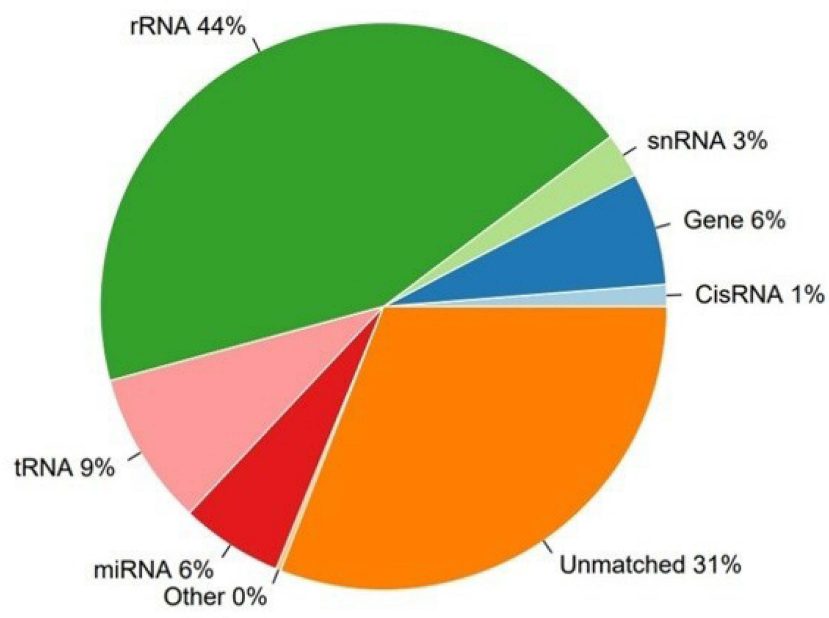

B

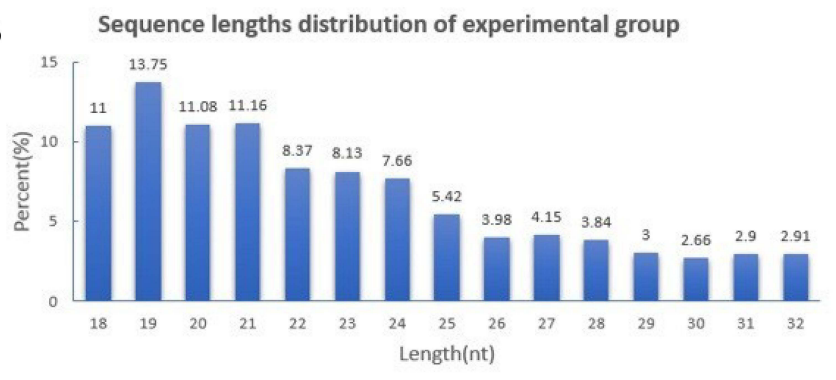

D

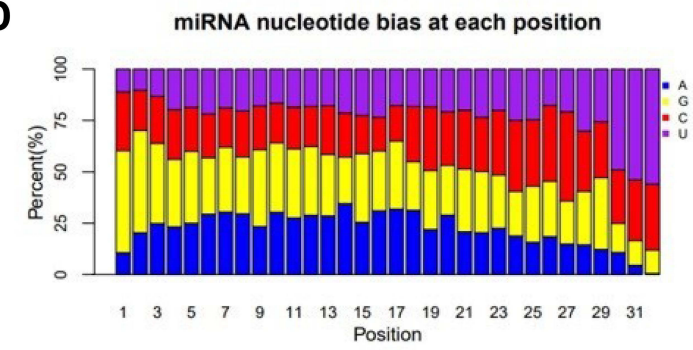

Mapped RNAs of experimental group

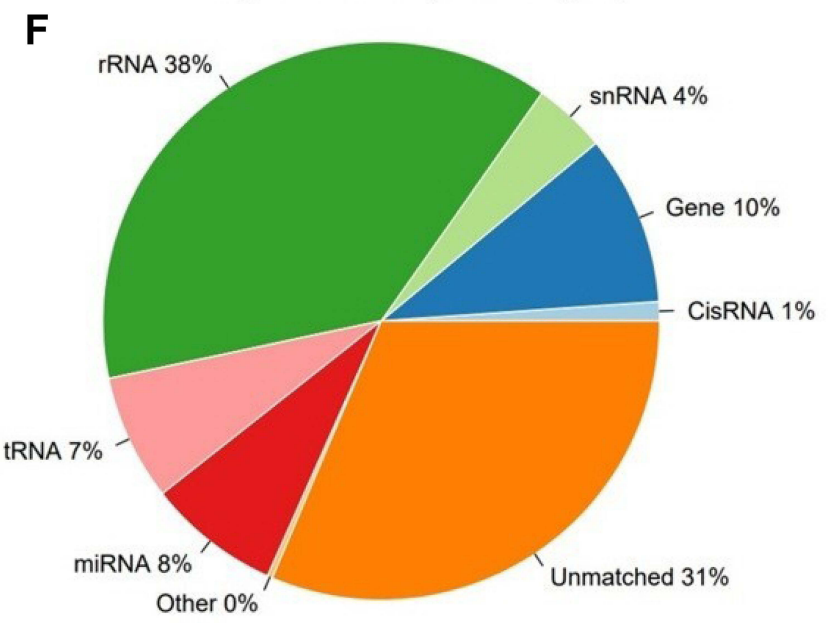

Figure 3 Characterization of exosome miRNAs. (A) Sequence lengths distribution of the control group. (B) Sequence lengths distribution of the experimental group. (C) miRNA nucleotide bias at each position of the control group. (D) miRNA nucleotide bias at each position of the experimental group. (E) Distribution of small RNA species in serum exosome of the control group. (F) Distribution of small RNA species in serum exosome of the experimental group.

revealed 73 known up-regulated miRNAs and 59 known down-regulated miRNAs (absolute value of $\log 2 \mathrm{FC} \geq 1$ and FDR $<0.01$ ) (Figure 5E and Table 3). These differentially expressed miRNAs were ranked according to the $\log 2 \mathrm{FC}$. The top ten up-regulated miRNAs were miR-5589-3p, miR194-3p, miR-378c, miR-556-3p, miR-4271, miR-141-3p, miR-148a-5p, miR-548h-3p, miR-548z and miR-497-5p (Figure 5C); meanwhile, the top ten down-regulated miRNAs were miR-205-5p, miR-18a-5p, miR-335-3p, miR-942-5p, miR-3605-5p, miR-379-3p, let-7e-3p, miR4433b-3p, miR-636, and miR-935 (Figure 5D).

\section{Validation of the RNA-sequencing Data by qRT-PCR Analysis}

According to the expression levels of miRNAs, 8 candidate miRNAs including 5 up-regulated and 3 downregulated miRNAs were analyzed in the smaller test cohort of 17 subjects in control group and 15 subjects in experimental group using qRT-PCR. The qRT-PCR results showed that miR-192-5p, miR-194-5p, miR-455-3p and miR-1246 were expressed at levels more than 1.5-fold higher while miR-199a-3p and miR-486-5p were less than 0.67 -fold lower in AIDS combined with T.M 


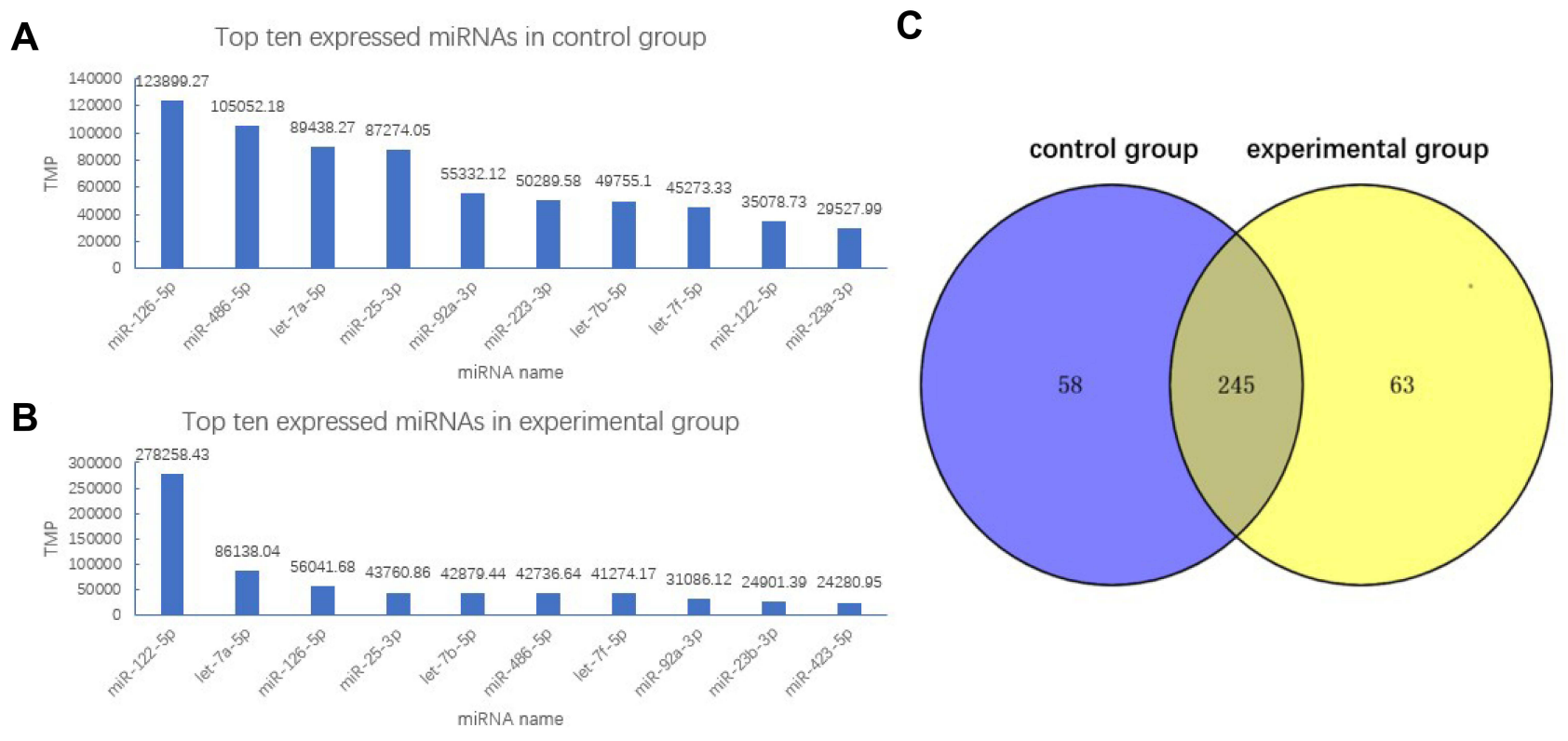

Figure 4 Small RNA-seq miRNA profiles in control and experimental groups. (A) The top 10 expressed miRNAs in the control group. (B) The top I0 expressed miRNAs in the experimental group. (C) Venn diagram showing the common and unique miRNAs identified in control and experimental groups.

infection patients (Table 4, Test cohort). To further validate, the expression levels of the four high expressed miRNAs in 35 subjects in control group and 33 subjects in experimental group, including previous smaller test cohort patients, were further analyzed. Based on validation results, the four miRNAs (miR-192-5p, miR-194-5p and miR-1246) were significantly upregulated in serum exosomes from AIDS combined with T.M infection patients (Fold change $\geq 2$ and p-value $<0.05$ ) (Figure 6, Table 4, validation cohort).

\section{Predictive Significance of Exosomal miR-192-5p, miR-194-5p and miR-I246 in the Diagnosis of AIDS Patients with T.M Infection}

Further, ROC curves were plotted to assess the diagnostic ability of these four miRNAs to identify AIDS patients with T.M infection. The area under the curve (AUC) values for miR-192-5p, miR-194-5p and miR-1246 were $0.658,0.703$ and 0.721 , respectively, indicating the potential to distinguish AIDS patients with T.M infection from AIDS patients without T.M infection (Figure 7A-C). The diagnostic sensitivity and specificity of miR-192-5p, miR194-5p and miR-1246 for AIDS patients with T.M infection were 0.676 and $0.611,0.649$ and $0.694,0.703$ and 0.75 , respectively (Table 5 ). Then, the combined diagnosis and analysis of 3 miRNAs were performed. The AUC of combined diagnosis was 0.742 , and the sensitivity and specificity of diagnosis were 0.568 and 0.861 , respectively. The AUC and specificity of the combined diagnosis for the three miRNAs were significantly improved (Figure 7D and Table 5).

\section{GO and KEGG Pathways}

In this experiment, Gene Ontology enrichment analysis was performed on the target genes of the three miRNAs, and the top 10 enrichment results were analyzed. The results showed that in the biological process analysis, all of the 3 miRNAs were involved in transcription, DNAtemplated and positive regulation of transcription from RNA polymerase II promoter. In addition, most of them were also involved in negative regulation of transcription from RNA polymerase II promoter, negative regulation of transcription, DNA-templated and positive regulation of transcription, DNA-templated (Figure 8A). This was consistent with molecular function enrichment results which were mainly involved protein binding, DNA binding, transcription factor activity, sequence-specific DNA binding, transcriptional corepressor activity, transcription regulatory region DNA binding, etc. (Figure 8B). Meanwhile, KEGG pathway analysis on the predicted targets led to the identification of significant pathways in which the predicted miRNA targets were enriched (Table 6) and the 


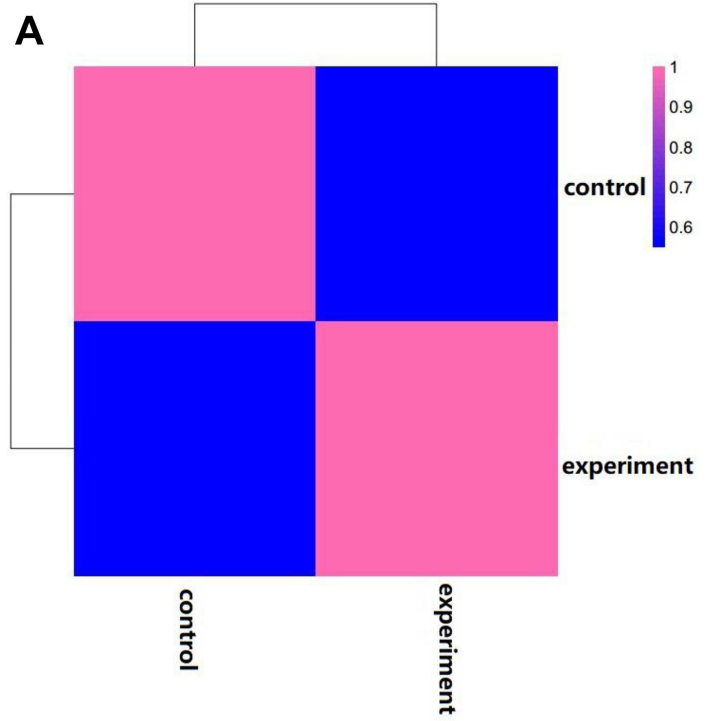

C The top ten up-regulated miRNAs

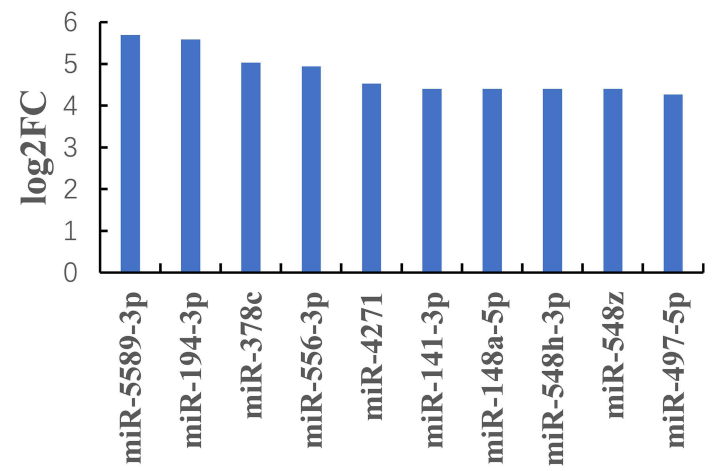

B

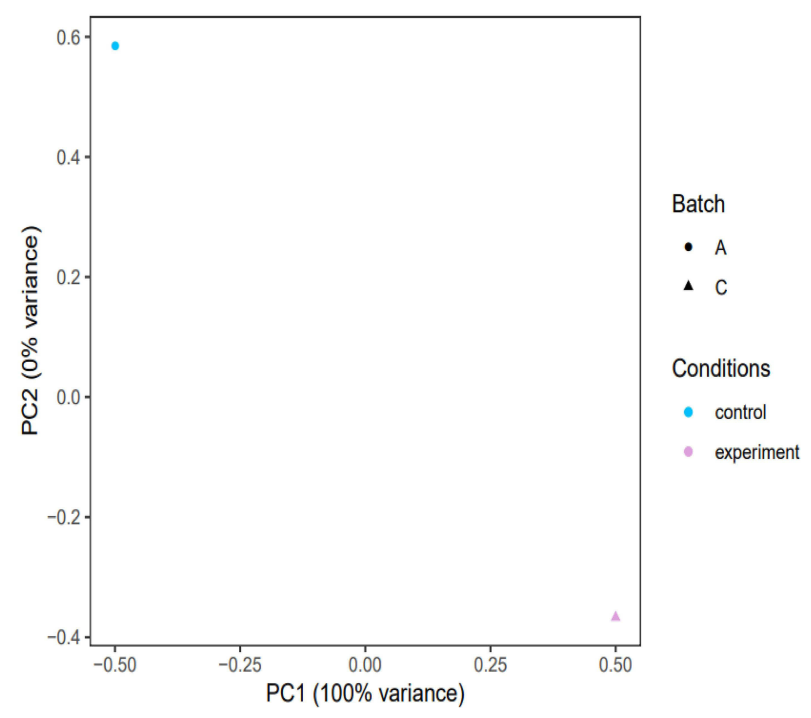

D The top ten down-regulated miRNAs

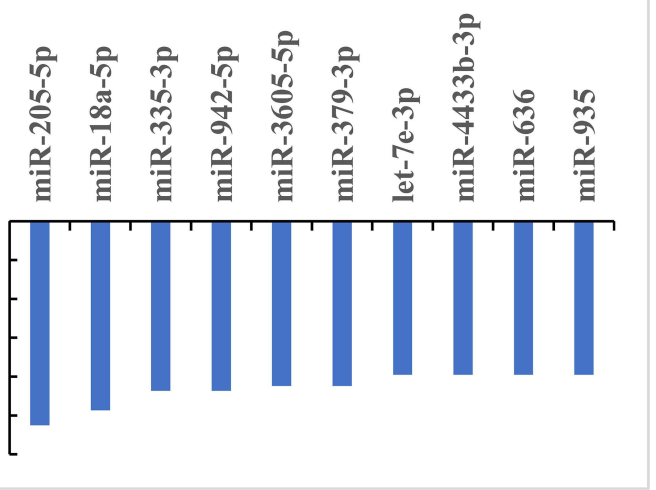

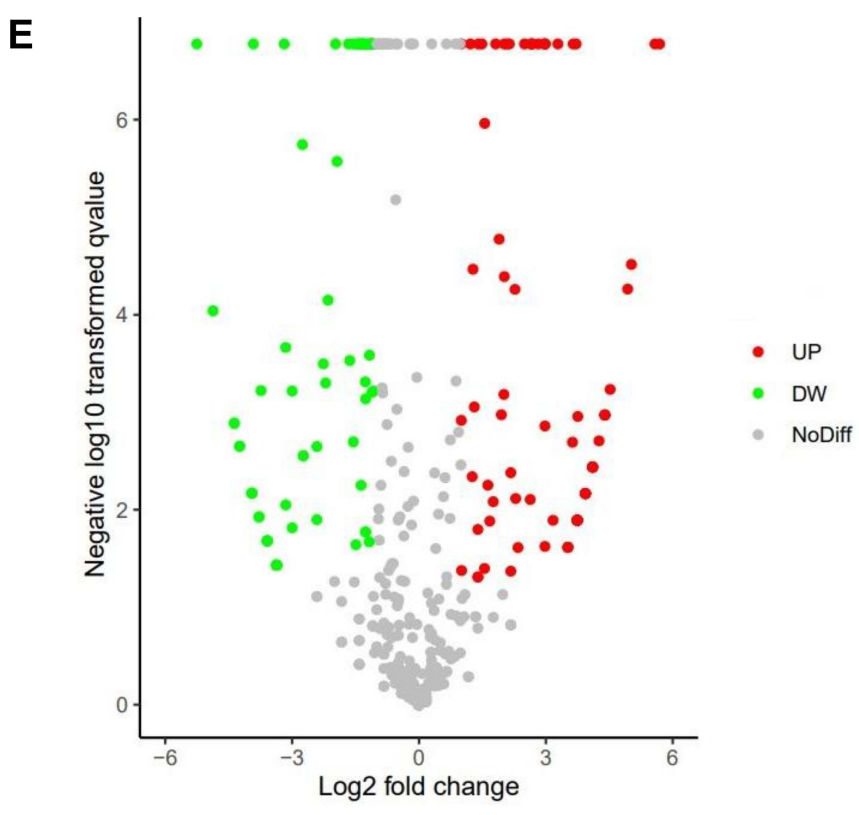

Figure 5 Differentially expressed exosomal miRNAs. (A) Cluster analysis of miRNAs in the two groups. (B) Principal component analysis of miRNAs. (C) The top 10 upregulated miRNAs in the experimental group. (D) The top 10 down-regulated miRNAs in the experimental group. (E) Volcano map showing the overall differentially expressed miRNAs (DEmiRNAs) in the two groups. Each point represents a miRNA. Points in green indicate downregulated miRNAs (log2FC $\geq I$ and FDR $<0.0 \mathrm{I}$ ), while points in red indicate upregulated miRNAs $(\log 2 F C \geq I$ and $F D R<0.0 I)$. Grey dots indicate miRNAs $(\log 2 F C<I$ and FDR $\geq 0.0 \mathrm{I})$. 
Table 3 Summary of Differentially Expressed miRNAs

\begin{tabular}{|c|c|}
\hline miRNA Counts & miRNA Names \\
\hline $\begin{array}{l}\text { Up-regulated miRNAs } \\
\text { (73) }\end{array}$ & $\begin{array}{l}\text { miR-5589-3p, miR-I94-3p, miR-556-3p, miR-427I, miR-I4I-3p, miR-I48a-5p, miR-548h-3p, miR-548z, miR-497-5p, let- } \\
\text { 7f-2-3p, miR-I34-5p, miR-200b-3p, miR-339-5p, miR-4433b-5p, miR-192-3p, miR-30b-3p, miR-4706, miR-500a-3p, miR- } \\
\text { 502-3p, miR-629-5p, miR-378i, miR-I294, miR-I8Ia-2-3p, miR-I83-3p, miR-30c-2-3p, miR-36I4-3p, miR-4306, miR- } \\
\text { 548ad-5p, miR-548ae-5p, miR-548ay-5p, miR-548d-5p, miR-6735-5p, miR-99a-5p, let-7i-3p, miR-I22-3p, miR-548aq-3p, } \\
\text { miR-455-3p, miR-9-3p, miR-I22-5p, miR-24-I-5p, miR-22-5p, miR-885-5p, miR-194-5p, miR-378a-3p, miR-365a-3p, miR- } \\
\text { 365b-3p, miR-94I, miR-192-5p, miR-380-3p, miR-378a-5p, miR-I468-5p, miR-574-3p, miR-505-3p, miR-23c, miR-23b- } \\
\text { 3p, miR-33I-3p, miR-382-5p, miR-222-3p, miR-I246, miR-802, miR-30Ia-3p, miR-204-5p, miR-I48a-3p, miR-483-5p, } \\
\text { miR-28-3p, miR-3605-3p, miR-I30b-5p, miR-2I5-5p, miR-28-5p, miR-I25b-5p, miR-27b-3p, miR-340-3p }\end{array}$ \\
\hline $\begin{array}{l}\text { Down-regulated } \\
\text { miRNAs (59) }\end{array}$ & 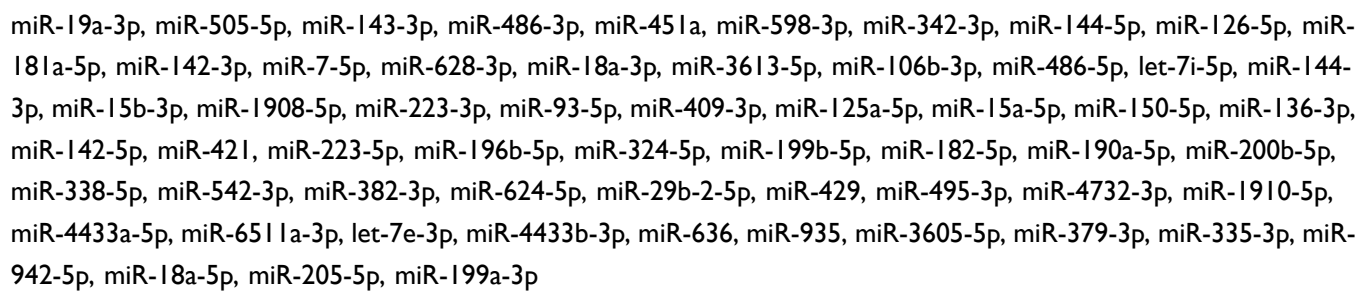 \\
\hline
\end{tabular}

Table 4 Relative Expression of Candidate miRNAs

\begin{tabular}{|l|c|c|c|c|}
\hline \multirow{2}{*}{ Gene Symbol } & \multicolumn{2}{|c|}{$\begin{array}{c}\text { Test Cohort (Control Group n= I 7; Experimental } \\
\text { Group n=1 5) }\end{array}$} & $\begin{array}{c}\text { Validation Cohort (Control Group n=35; Experimental } \\
\text { Group } \mathbf{n = 3 3 )}\end{array}$ \\
\cline { 2 - 5 } & Fold Change & p-value & Fold Change & p-value \\
\hline miR-28-3p & 0.45 & 0.047 & 1.41 & 0.059 \\
miR-192-5p & 1.76 & 0.835 & 2.71 & $\mathbf{0 . 0 2}$ \\
miR-194-5p & 2.15 & 0.439 & 3.16 & $\mathbf{0 . 0 0 3}$ \\
miR-455-3p & 2.04 & 0.895 & 2.27 & 0.604 \\
miR-1246 & 2.21 & 0.168 & 5.48 & $\mathbf{0 . 0 0 1}$ \\
miR-144-3p & 0.98 & 0.302 & 1.51 & 0.674 \\
miR-199a-3p & 0.57 & 0.336 & 1.33 & 0.856 \\
miR-486-5p & 0.24 & 0.001 & 0.53 & 0.057 \\
\hline
\end{tabular}

Note: Bold in the table indicates important data.

top ten pathways of the three miRNAs are listed in Figure 9. Specifically, miRNA targets associated with AIDS with T.M infection belonged to multiple pathways such as pathways in TGF-beta signaling pathway, AMPK signaling pathway, cAMP signaling pathway, Wnt signaling pathway and MAPK signaling pathway, etc. (Table 6).

\section{Discussion}

HIV-1 is known to cause dramatic changes in circulating miRNA $^{22}$ and milk derived exosomal miRNA ${ }^{23}$ expression profiles, however its effect on serum exosomal miRNAs derived from TM infection patients remains unknown. Here, we characterized miRNA expression profiles of serum exosomes derived from AIDS patients infected TM and showed that HIV-1 with TM infection significantly altered expression levels of exosomal miRNAs. Analysis of differentially expressed miRNAs by GO and KEGG pathway-based approach revealed several biological processes are affected by AIDS combined with TM infection. Furthermore, we identified four upregulated miRNAs that can be potentially discriminate AIDS combined with TM infection from uninfected $\mathrm{TM}$, with good predictive power. Collectively, these data provide, for the first time, comprehensive insight into serum exosomal miRNA profiles involved during AIDS combined with TM infection.

In this experiment, samples were selected strictly in accordance with the selection criteria. The final test cohort included 15 people in the experimental group and 17 people in the control group while the validation cohort included 33 people in the experimental group and 35 
A

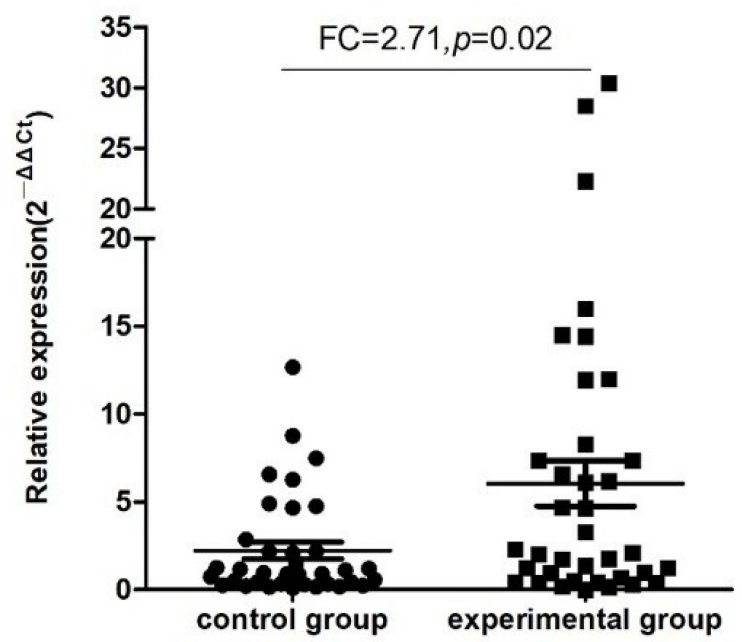

B

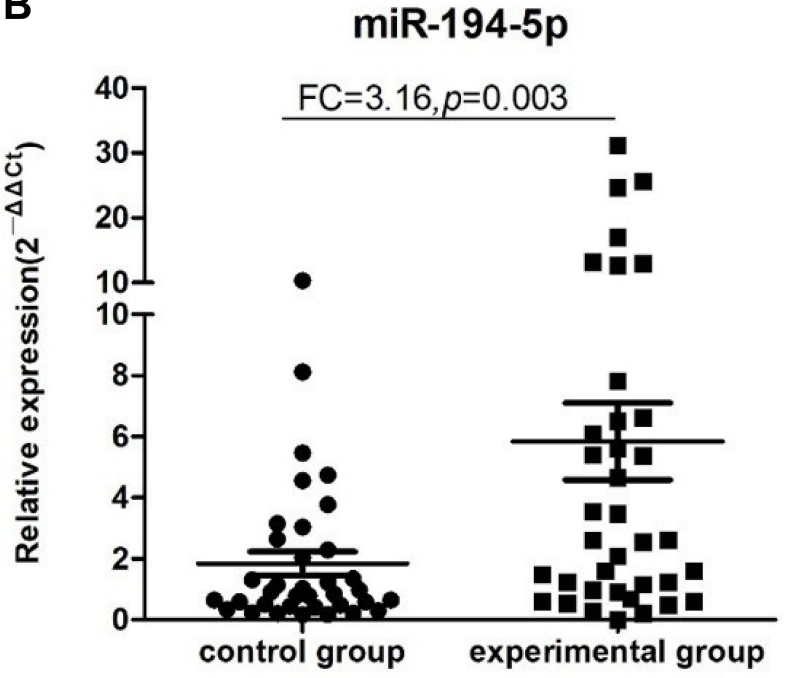

C

miR-1246

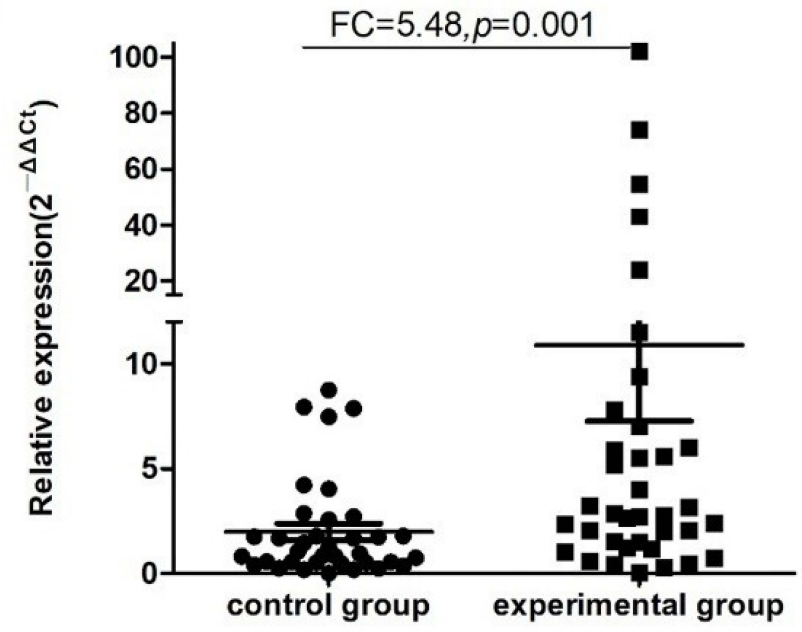

Figure 6 Validation of 4 high expressed miRNAs using qRT-PCR analysis. (A) Relative expression levels of miR-192-5p. (B) Relative expression levels of miR-194-5p. (C) Relative expression levels of miR-1246.

people in the control group, and the number of people in the test cohort was included in the validation cohort. The subjects included in the experimental group and the control group had no significant differences in age and gender, white blood cell, neutrophil, lymphocyte, and platelet counts. The monocyte count, $\mathrm{CD}^{+}{ }^{+} \mathrm{T}$ lymphocyte count, $\mathrm{CD}^{+} \mathrm{T}$ lymphocyte count, and the ratio of $\mathrm{CD} 4 / \mathrm{CD} 8$ in the experimental group were lower than those in the control group, but this is related to the clinical characteristics of the patients in the experimental group ${ }^{24,25}$ and this feature is inevitable in AIDS patients with TM infection. In addition, once TM invaded the human body and caused a systemic disseminated infection, it will first cause a mononuclear-macrophage system reaction, ${ }^{26}$ and that may be responsible for the decrease in the number of monocytes of AIDS combined with TM infection. The reduced $\mathrm{T}$ lymphocyte count and monocyte count in patients with AIDS co-infected with TM may affect the abundance, size and miRNA expression of exosomes. ${ }^{18}$ This is also the reason why we will explore the early markers of serum exosomes in AIDS combined with TM infection.

The current traditional methods for diagnosing TM cannot perform early and rapid diagnosis of TM, and the identification of new potential biomarkers is urgent. To gain more insight into serum exosomal miRNAs 
A

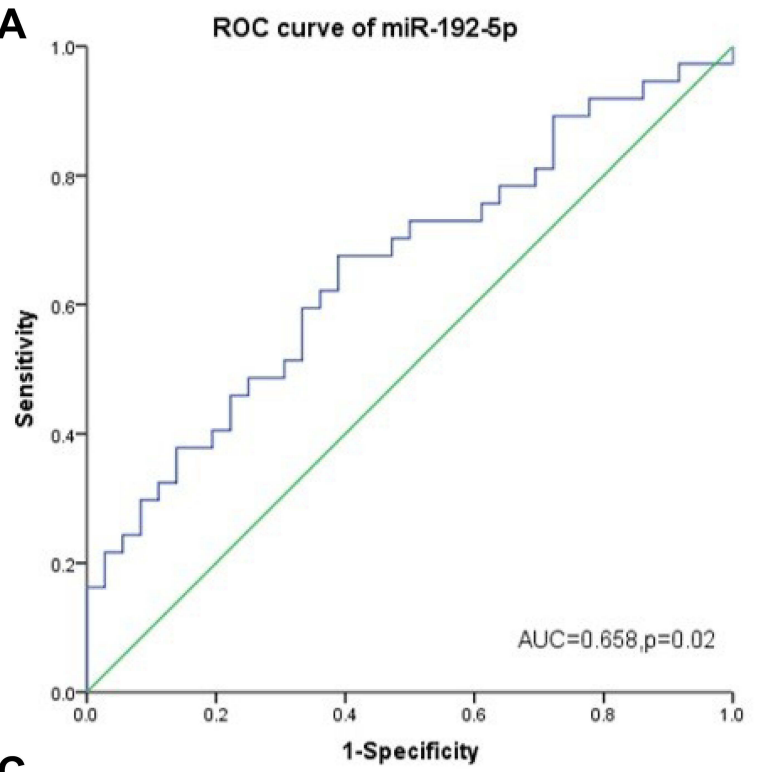

C

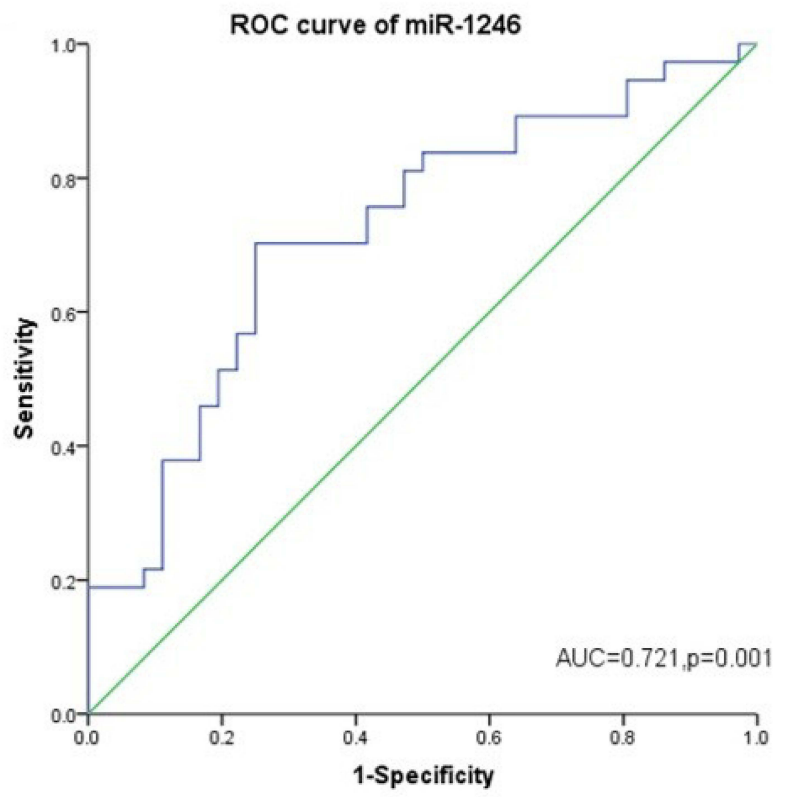

B

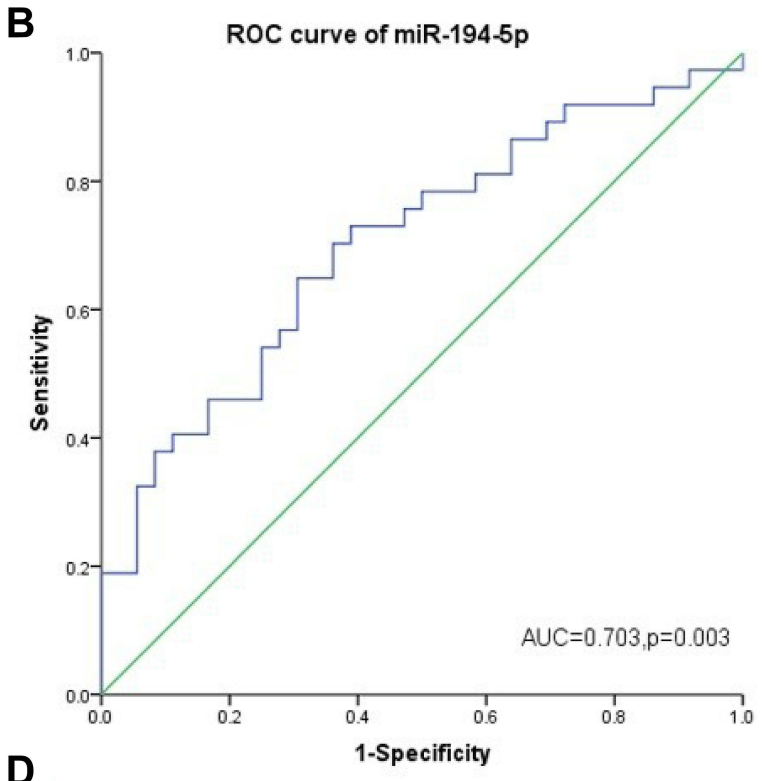

D

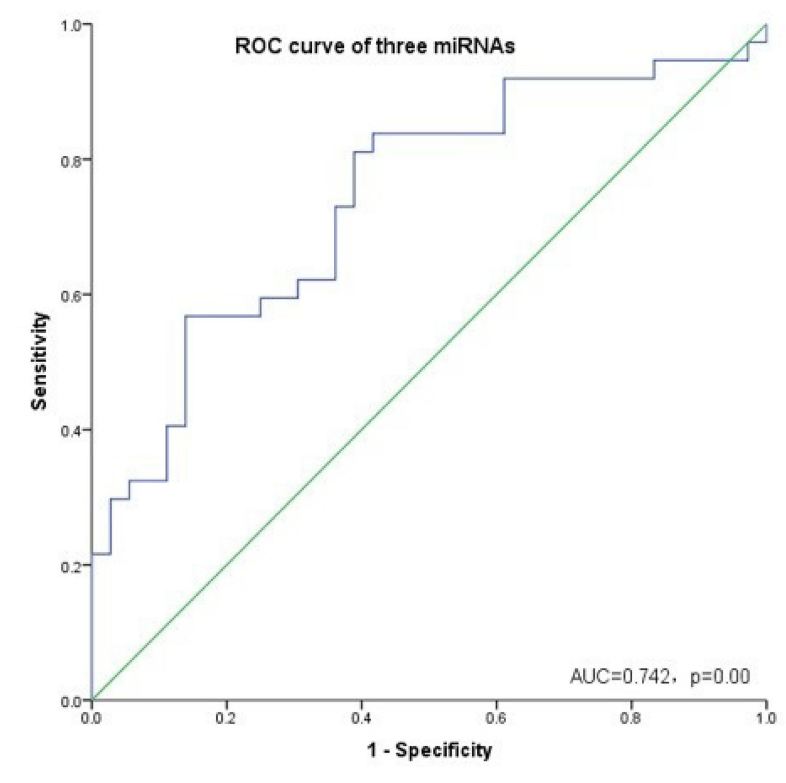

Figure 7 ROC curve of 4 high expressed miRNAs. (A) ROC curve of miR-192-5p. (B) ROC curve of miR-194-5p. (C) ROC curve of miR-1246. (D) ROC curve of the three miRNAs for combined diagnosis.

modulation by AIDS combined with TM infection, we performed serum exosomal miRNA profiling and showed that serum exosomes derived from AIDS combined with

Table 5 Diagnostic Sensitivity and Specificity of the Four miRNAs

\begin{tabular}{|l|l|l|l|}
\hline miRNA & AUC & Sensitivity & Specificity \\
\hline miR-192-5p & 0.658 & 0.676 & 0.611 \\
miR-194-5p & 0.703 & 0.649 & 0.694 \\
miR-1246 & 0.721 & 0.703 & 0.75 \\
Combined diagnosis of 3 miRNAs & 0.742 & 0.568 & 0.861 \\
\hline
\end{tabular}

TM infection perturbed the expression levels of 131 miRNAs (FC $>2, P<0.01$, Table 3 ). The combination of three miRNAs (miR-192-5p, miR-194-5p and miR-1246) had AUC of 0.742 , a sensitivity of $56.8 \%$ and a specificity of $86.1 \%$ in predicting AIDS combined with TM infection. And in previous literature reports, these three miRNAs were closely related to the body's inflammatory response, immune response, and disease progression. ${ }^{27-30}$ Except that, as a cell endopathogenic fungi, the immune clearance mechanism of TM infected host involves innate immunity and specific immune system, so the up-regulated miR-192- 


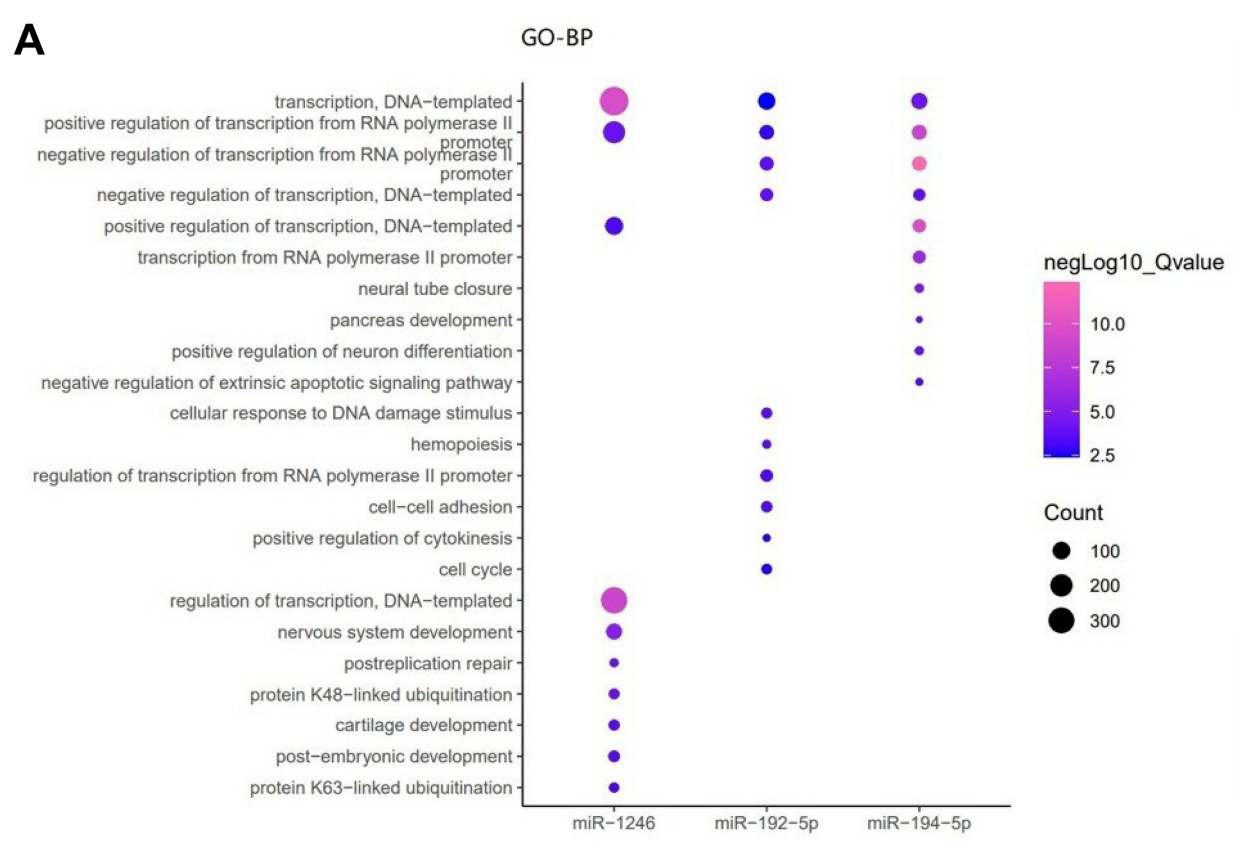

B

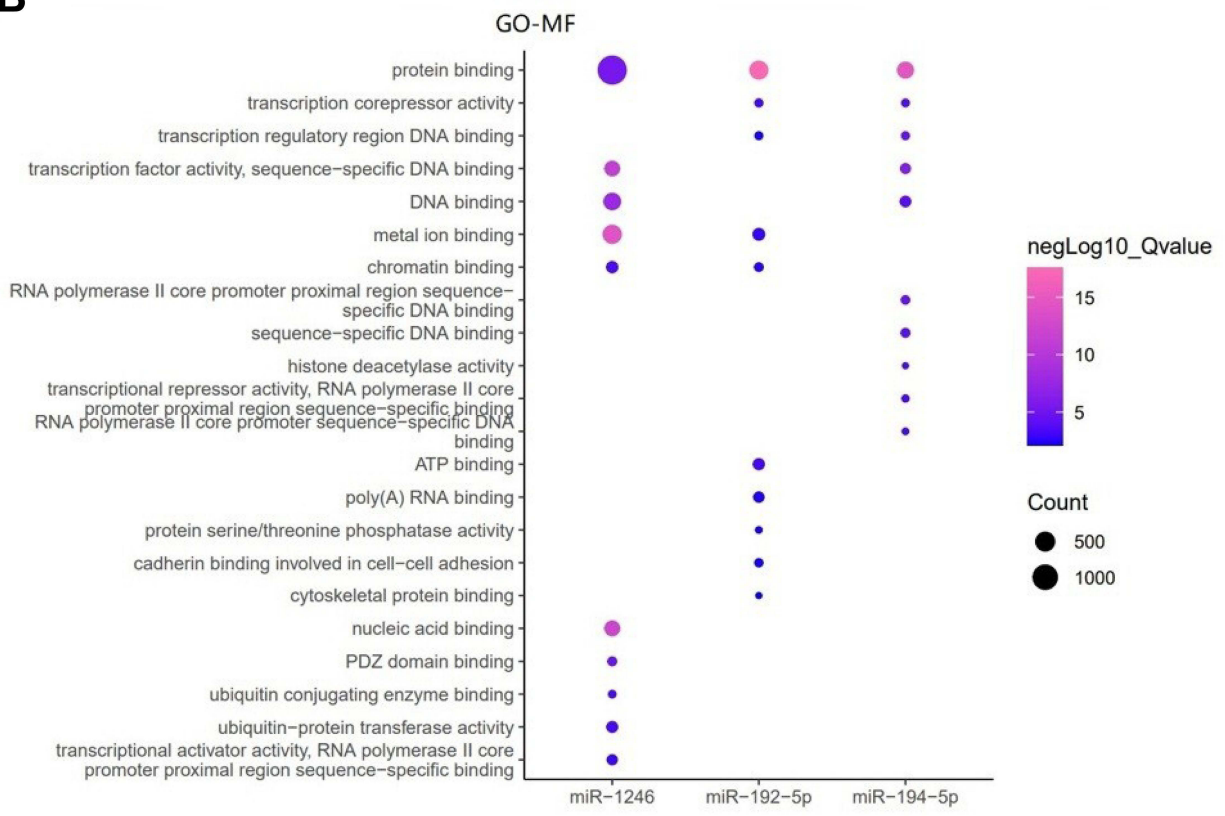

Figure 8 Gene Ontology enrichment analysis of the three miRNAs. (A) Target-related Gene Ontology (GO) enrichment results in biological process of the three miRNAs. (B) Target-related Gene Ontology (GO) enrichment results in molecular function of the three miRNAs.

$5 \mathrm{p}, \mathrm{miR}-194-5 \mathrm{p}$ and miR-1246 were verified in this experiment may have a certain diagnostic potential for patients with AIDS combined with TM, and may become a potential non-invasive biomarker for patients with AIDS combined with TM infection.

Further, the pathway enrichment results showed that the pathways involved in the four miRNAs ranged from 8 to 30 , and the pathways related to cancer, diabetes, and nervous system, etc. were eliminated. The results showed that the regulation of the TGF- $\beta$ signaling pathway was related to miR-192-5p and miR-194-5p. In addition, miR-192-5p was also involved in AMPK signaling pathway, miR-1246 was significantly related to cGMP-PKG and cAMP signaling pathways, miR-194-5p may regulate Wnt and MAPK signaling pathways. Among them, AMPK signaling pathway had been found to be associated with infection in previous reports. ${ }^{31,32}$ In terms of HIV-1 infection, it had been reported that HIV-1 infection leaded to a sharp decrease in the 
Table 6 Pathways of Three miRNAs Enrichment

\begin{tabular}{|c|c|c|c|}
\hline miRNA Names & Description & Qvalue & Count \\
\hline \multirow[t]{8}{*}{ miR-192-5p (8) } & Insulin signaling pathway & 0.005152 & 13 \\
\hline & p53 signaling pathway & 0.011797 & 8 \\
\hline & TGF-beta signaling pathway & 0.012504 & 9 \\
\hline & Signaling pathways regulating pluripotency of stem cells & 0.035495 & II \\
\hline & Small cell lung cancer & 0.038283 & 8 \\
\hline & AMPK signaling pathway & 0.039336 & 10 \\
\hline & Cell cycle & 0.041095 & 10 \\
\hline & Dopaminergic synapse & 0.048664 & 10 \\
\hline \multirow[t]{26}{*}{ miR-194-5p (26) } & Ubiquitin mediated proteolysis & $4.9 \mathrm{E}-05$ & 14 \\
\hline & TGF-beta signaling pathway & $5.5 \mathrm{E}-05$ & 11 \\
\hline & Axon guidance & 0.0001 & 13 \\
\hline & Pathways in cancer & 0.000473 & 23 \\
\hline & Wnt signaling pathway & 0.000849 & 12 \\
\hline & Signaling pathways regulating pluripotency of stem cells & 0.000957 & 12 \\
\hline & ErbB signaling pathway & 0.001757 & 9 \\
\hline & MAPK signaling pathway & 0.002237 & 16 \\
\hline & Focal adhesion & 0.002547 & 14 \\
\hline & Glioma & 0.006438 & 7 \\
\hline & Renal cell carcinoma & 0.006934 & 7 \\
\hline & Regulation of actin cytoskeleton & 0.008095 & 13 \\
\hline & Prolactin signaling pathway & 0.009833 & 7 \\
\hline & HIF-I signaling pathway & 0.011898 & 8 \\
\hline & Basal cell carcinoma & 0.012455 & 6 \\
\hline & Proteoglycans in cancer & 0.014413 & 12 \\
\hline & Rap I signaling pathway & 0.020032 & 12 \\
\hline & Insulin signaling pathway & 0.026359 & 9 \\
\hline & Transcriptional misregulation in cancer & 0.028898 & 10 \\
\hline & Hedgehog signaling pathway & 0.031596 & 4 \\
\hline & Neurotrophin signaling pathway & 0.035608 & 8 \\
\hline & Melanoma & 0.036195 & 6 \\
\hline & Chronic myeloid leukemia & 0.038112 & 6 \\
\hline & Glucagon signaling pathway & 0.042785 & 7 \\
\hline & Melanogenesis & 0.04458 & 7 \\
\hline & Choline metabolism in cancer & 0.046421 & 7 \\
\hline
\end{tabular}

(Continued)

cellular peroxidase pool, and Wnt signaling played an important role in peroxisome homeostasis by regulating the production of biogenesis factors, ${ }^{33}$ suggesting that Wnt signaling was closely related to HIV-1, and miR-194-5p as a candidate marker for AIDS combined infected with TM, may be involved in the process of Wnt signaling pathway.

Except that, the pathways that may be associated with AIDS and TM infections enriched by miR-1246 included gap junctions, cAMP signaling pathways and cGMP-PKG signaling pathways. As we know, the miRNA can be transferred from the original cell to its neighboring cells, and had an active function in the recipient cell. ${ }^{17}$ Meanwhile, the transfer of miRNA involved intercellular gap junctions. Therefore, it can be inferred that there may be a close relationship between changes in serum exosomes miR-1246 and gap junctions in AIDS combined with TM infected patients. In addition, the immune response is an important physiological response to infection. A recent report revealed the potential regulatory effect of exosomal miRNA in the plasma of patients with sepsis on the immune system. ${ }^{34}$ On this basis, some scholars $^{35}$ confirmed that exosomes derived from sepsis pass miR-7 -5 p changed the mRNA and protein levels of apoptosis-related genes, thereby regulating the apoptosis of $\mathrm{T}$ lymphocytes. The double luciferase reporter gene detection method confirmed that miR-7-5p played 
Table 6 (Continued).

\begin{tabular}{|c|c|c|c|}
\hline miRNA Names & Description & Qvalue & Count \\
\hline miR-I246 (30) & $\begin{array}{l}\text { Proteoglycans in cancer } \\
\text { Signaling pathways regulating pluripotency of stem cells } \\
\text { Pathways in cancer } \\
\text { Salivary secretion } \\
\text { Ras signaling pathway } \\
\text { Regulation of actin cytoskeleton } \\
\text { Insulin signaling pathway } \\
\text { Dopaminergic synapse } \\
\text { Retrograde endocannabinoid signaling } \\
\text { Long-term potentiation } \\
\text { Neurotrophin signaling pathway } \\
\text { Gap junction } \\
\text { Estrogen signaling pathway } \\
\text { Adrenergic signaling in cardiomyocytes } \\
\text { Gastric acid secretion } \\
\text { Melanogenesis } \\
\text { cAMP signaling pathway } \\
\text { Insulin resistance } \\
\text { Long-term depression } \\
\text { Thyroid hormone signaling pathway } \\
\text { Transcriptional misregulation in cancer } \\
\text { Hippo signaling pathway } \\
\text { cGMP-PKG signaling pathway } \\
\text { Circadian entrainment } \\
\text { Ubiquitin mediated proteolysis } \\
\text { Aldosterone synthesis and secretion } \\
\text { Thyroid hormone synthesis } \\
\text { Cholinergic synapse } \\
\text { Prostate cancer } \\
\text { Vascular smooth muscle contraction } \\
\text { ald }\end{array}$ & $\begin{array}{l}3.39 \mathrm{E}-04 \\
0.002358 \\
0.00337 \\
0.004264 \\
0.005009 \\
0.005646 \\
0.006715 \\
0.007582 \\
0.007889 \\
0.010477 \\
0.010713 \\
0.011861 \\
0.012089 \\
0.012134 \\
0.013617 \\
0.013629 \\
0.014808 \\
0.018005 \\
0.020478 \\
0.020805 \\
0.020972 \\
0.023909 \\
0.026338 \\
0.027231 \\
0.032395 \\
0.037146 \\
0.037734 \\
0.042819 \\
0.042909 \\
0.043484\end{array}$ & $\begin{array}{l}48 \\
34 \\
77 \\
23 \\
48 \\
45 \\
32 \\
30 \\
25 \\
18 \\
28 \\
22 \\
24 \\
31 \\
19 \\
24 \\
41 \\
25 \\
16 \\
26 \\
35 \\
32 \\
33 \\
22 \\
29 \\
19 \\
17 \\
24 \\
20 \\
25\end{array}$ \\
\hline
\end{tabular}

a negative regulatory role on the bad gene (a well-known pro-apoptotic gene) in the cGMP-PKG signaling pathway. It can be seen that the cGMP-PKG signaling pathway was very likely to participate in the inflammatory regulation process of miRNA. Except that, as a kind of cyclic nucleotide system, cAMP signaling pathway is also called protein kinase A (PKA) system. In this system, extracellular signals can be combined with corresponding receptors, and caused a response by regulating the level of intracellular second messenger cAMP. Signal molecules can be inflammatory factors, pathogens and their products, hormones, etc. The regulation of cAMP was carried out by adenylate cyclase, and cAMP regulates key physiological processes, including metabolism, secretion, cell fate and gene transcription. In some studies, ${ }^{36}$ caffeine was shown to reduce the expression of miR-301b through the negative regulation of the cAMP/PKA/NF- $\mathrm{BB}$ axis, thereby enhancing respiratory immunity and inhibiting the inflammatory response during lung infection. In addition, some research $^{37}$ found that miR-150 can inhibit the activity of PI3K and AKT through the cAMP-PKA-Csk signaling pathway, thereby effectively preventing CD28/B7 costimulatory signal transduction, reducing inflammatory cytokines (such as IL-2 and TNF) and inducing immune tolerance.

Finally, the TGF- $\beta$ signaling pathway related to miR192-5p and miR-194-5p plays an important role in the development, homeostasis and repair of most tissues in the organism. All immune cell lineages, including B cells, T lymphocytes, dendritic cells and macrophages, can secrete TGF- $\beta$. It can negatively regulate cell proliferation, differentiation and activation of other cytokines. 


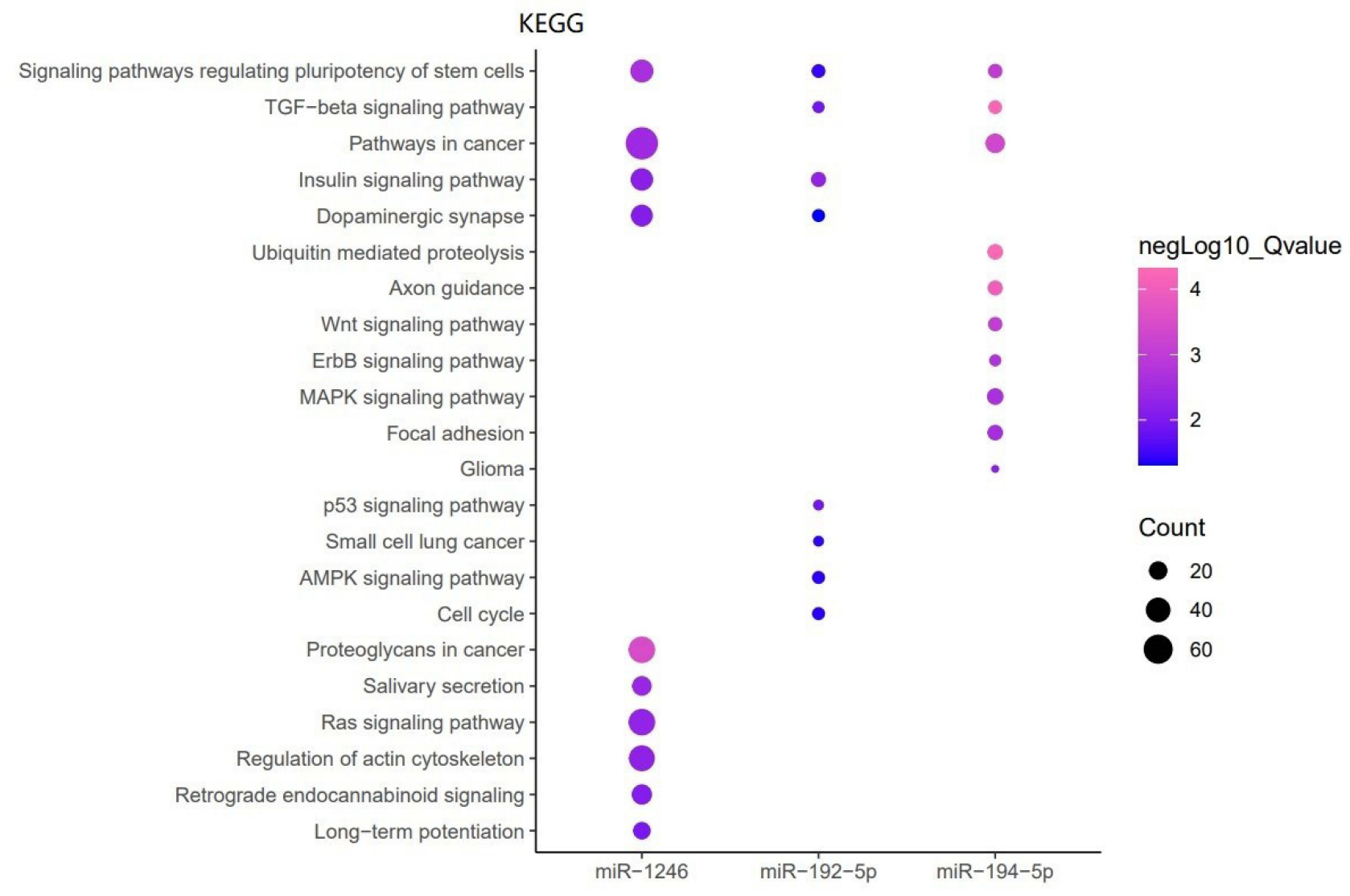

Figure 9 The top ten pathways of the three miRNAs enriched by KEGG.

Related reports had shown that the miRNA-enriched pathway associated with HIV-1 infection involved the TGF- $\beta$ signaling pathway. ${ }^{22,23}$ In terms of inflammation, some scholars $^{38}$ compared exosomes derived from human amniotic epithelial cells with human lungs. Comparing the protein and miRNA content of fibroblast exosomes, it was found that exosomes derived from human amniotic epithelial cells can increase the phagocytosis of macrophages, reduce neutrophil myeloperoxidase, and directly inhibit T Lymphocytes proliferate and reduce lung inflammation, while exosomal miRNA derived from human amniotic epithelial cells was enriched in TGF- $\beta$ and other pathways. In addition, in many fungal diseases, dendritic cells (DC), which promote inflammation and tolerance, were involved in the regulation of $\mathrm{CD}^{+}$ $\mathrm{T}$ lymphocyte response, and TM infection also led to an increase in expression of CD80 and CD86 on DC. This also caused a significant increase in the level of TGF- $\beta$ in the cell supernatant, ${ }^{39}$ suggesting that TM infection was closely related to TGF- $\beta$ signal transduction. It can be seen that TGF- $\beta$ signaling was an inflammatory signaling pathway, which was involved in the regulation of exosomal miRNAs derived from cells infected by bacteria ${ }^{38}$ and fungi. ${ }^{39}$ In our research, the differentially expressed exosomal miR-192-5p and miR-194-5p derived from the patients with AIDS combined with TM infection may all be involved in the TGF- $\beta$ signal transduction pathway.

In summary, the multiple pathways enriched by miR192-5p, miR-194-5p and miR-1246, especially the TGF- $\beta$ signal pathway enriched by miR-192-5p and miR-194-5p, may be involved in processes such as inflammation and immune response, which were consistent with the biological functions of exosomes, and also indicated that this study was in the serum exosomes of patients for AIDS combined with TM infection. The verified miRNA alteration mechanism may involve these infected signaling pathways, and the specific targeted pathways need to be further studied.

\section{Conclusions}

In conclusion, our study shows that serum exosomal miRNAs of patients with AIDS and TM infection have 
changed significantly. Through miRNA sequencing and qRT-PCR validation studies, the results showed that three miRNAs (miR-192-5p, miR-194-5p and miR-1246) were significantly elevated in serum exosomes of AIDS patients with TM infection, and these miRNAs correlated with inflammatory response, immune response, and disease progression. These upregulated serum exosomal miRNAs are predicted to have functional associations with TGF- $\beta$ signaling pathway, AMPK signaling pathway, Wnt signaling pathway, MAPK signaling pathway, cGMP-PKG signaling pathway and cAMP signaling pathway, etc. These findings suggest that serum exosomal miRNAs may reflect ongoing pathophysiological processes in AIDS combined with TM infection, serving as potential biomarkers of AIDS combined with TM infection and targetable mechanisms involved in disease pathogenesis.

This study is the first time to explore the serum exosomes of patients with AIDS and TM infection. In the experiment, we combined the analysis method of bioinformatics, and the excavated miRNAs that are differentially expressed in the serum of people infected with AIDS and TM have certain diagnostic value. Some limitations in our study include the sample size, the lack of healthy controls and the fact that this was a simple cross-sectional study design. Further investigation about miR-192-5p, miR-194$5 p$ and miR-1246 and the relationships among these three miRNAs may be useful for the early diagnosis in AIDS combined with TM infection patients. In addition, further research with a large number of samples, the pathways and target genes of miRNA deduced by GO and KEGG and additional miRNAs in known pathways is also warranted.

\section{Acknowledgments}

We are grateful to all the participants who made this research possible and thank Guangxi Key Laboratory of AIDS Prevention and Treatment for providing the experimental platform.

\section{Funding}

This research was supported by Innovation Project of Guangxi Graduate Education (YCBZ2020046), Guangxi First-class Discipline Project for Basic medicine Sciences (GXFCDP-BMS-2019), Key R\&D projects in Nanning (20193008-1) and National Natural Science Foundation of China (31560050).

\section{Disclosure}

The authors declare no conflicts of interest in this work.

\section{References}

1. World Health Organization. HIV data and statistics. Available from: https://www.who.int/hiv/data/en/. Accessed November 8, 2021.

2. China Center for Disease Control and Prevention; 2020 (updated April 20; cited May 1, 2020). Available from: http://www.nhc.gov. $\mathrm{cn} / \mathrm{jkj} / \mathrm{s3578/202004/b1519e1bc1a944fc8ec176db600f68d1.shtml.}$ Accessed November 11, 2021.

3. Qiao YC, Xu Y, Jiang DX, et al. Epidemiological analyses of regional and age differences of HIV/AIDS prevalence in China, 2004-2016. Int $J$ Infect Dis. 2019;81:215-220. doi:10.1016/j.ijid.2019.02.016

4. Wang Y, Yang Y, Shi X, et al. The spatial distribution pattern of human immunodeficiency virus/acquired immune deficiency syndrome in China. Geospat Health. 2016;11(2):414. doi:10.4081/ gh.2016.414

5. Deng Z, Ribas JL, Gibson DW, et al. Infections caused by Penicillium marneffei in China and Southeast Asia: review of eighteen published cases and report of four more Chinese cases. Rev Infect Dis. 1988;10(3):640-652. doi:10.1093/clinids/10.3.640

6. Hu Y, Zhang J, Li X, et al. Penicillium marneffei infection: an emerging disease in mainland China. Mycopathologia. 2013;175(12):57-67. doi:10.1007/s11046-012-9577-0

7. Jianglong Qin YQ, Yibo L, Boming L. The epidemiology of Talaromyces marneffei infection in HIV-infected patients in Guangxi. China Trop Med. 2019;19(9):896-899.

8. Liu B, Fu P. Research progress of Penicilliosis marneffei. J Dermatol Venereol. 2010;32:26-28.

9. Delabranche X, Berger A, Boisramé-Helms J, et al. Microparticles and infectious diseases. Med Mal Infect. 2012;42(8):335-343. doi:10.1016/j.medmal.2012.05.011

10. Laganà A, Russo F, Veneziano D, et al. Extracellular circulating viral microRNAs: current knowledge and perspectives. Front Genet. 2013;4:120. doi:10.3389/fgene.2013.00120

11. Corrado C, Raimondo S, Chiesi A, et al. Exosomes as intercellular signaling organelles involved in health and disease: basic science and clinical applications. Int $J$ Mol Sci. 2013;14(3):5338-5366. doi:10.3390/ijms14035338

12. Lee RC, Feinbaum RL, Ambros V. The C. elegans heterochronic gene lin-4 encodes small RNAs with antisense complementarity to lin-14. Cell. 1993;75(5):843-854. doi:10.1016/0092-8674(93) 90529-Y

13. Lau SK, Chow WN, Wong AY, et al. Identification of microRNA-like RNAs in mycelial and yeast phases of the thermal dimorphic fungus Penicillium marneffei. PLoS Negl Trop Dis. 2013;7(8):e2398. doi:10.1371/journal.pntd.0002398

14. Li Y, Wei W, An S, et al. Identification and analysis of lncRNA, microRNA and mRNA expression profiles and construction of ceRNA network in Talaromyces marneffei-infected THP-1 macrophage. Peer J. 2021;9:e10529. doi:10.7717/peerj.10529

15. Li Y, Liu G, Zhang J, et al. Identification of key genes in human airway epithelial cells in response to respiratory pathogens using microarray analysis. BMC Microbiol. 2018;18(1):58. doi:10.1186/ s12866-018-1187-7

16. Pegtel DM, Cosmopoulos K, Thorley-Lawson DA, et al. Functional delivery of viral miRNAs via exosomes. Proc Natl Acad Sci USA. 2010;107(14):6328-6333. doi:10.1073/pnas.0914843107

17. Valadi H, Ekstrom K, Bossios A, et al. Exosome-mediated transfer of mRNAs and microRNAs is a novel mechanism of genetic exchange between cells. Nat Cell Biol. 2007;9(6):654-659. doi:10.1038/ ncb1596

18. Hubert A, Subra C, Jenabian MA, et al. Elevated abundance, size, and microRNA content of plasma extracellular vesicles in viremic HIV-1+ patients: correlations with known markers of disease progression. J Acquir Immune Defic Syndr. 2015;70(3):219-227. doi:10.1097/QAI.0000000000000756 
19. ExoQuick ${ }^{\circledR}$ Exosome isolation and RNA purification kits; 2018. Available from: https://systembio.com/shop/exoquick-exosome-rnapurification-kit-for-serum-plasma/. Accessed November 8, 2021.

20. Gagliardi A, Voci S, Salvatici MC, et al. Brij-stabilized zein nanoparticles as potential drug carriers. Colloids Surf B Biointerfaces. 2021;201:111647. doi:10.1016/j.colsurfb.2021.111647

21. Mir-X miRNA first-strand synthesis and TB green qRT-PCR user manual. Available from:https://www.takarabio.com/. Accessed November 8, 2021.

22. Chettimada S, Lorenz DR, Misra V, et al. Small RNA sequencing of extracellular vesicles identifies circulating miRNAs related to inflammation and oxidative stress in HIV patients. BMC Immunol. 2020;21 (1):57. doi:10.1186/s12865-020-00386-5

23. Zahoor MA, Yao XD, Henrick BM, et al. Expression profiling of human milk derived exosomal microRNAs and their targets in HIV-1 infected mothers. Sci Rep. 2020;10(1):12931. doi:10.1038/s41598020-69799-x

24. Sun J, Sun W, Tang Y, et al. Clinical characteristics and risk factors for poor prognosis among HIV patients with Talaromyces marneffei bloodstream infection. BMC Infect Dis. 2021;21(1):514. doi:10.1186/ s12879-021-06232-2

25. Li YY, Dong RJ, Shrestha S, et al. AIDS-associated Talaromyces marneffei central nervous system infection in patients of southwestern China. AIDS Res Ther. 2020;17:26.

26. Pongpom M, Vanittanakom $\mathrm{P}$, Nimmanee $\mathrm{P}$, et al. Adaptation to macrophage killing by Talaromyces marneffei. Future Sci OA. 2017;3(3):FSO215. doi:10.4155/fsoa-2017-0032

27. Liu XL, Pan Q, Cao HX, et al. Lipotoxic hepatocyte-derived exosomal microRNA 192-5p activates macrophages through Rictor/Akt/ Forkhead box transcription factor O1 signaling in nonalcoholic fatty liver disease. Hepatology. 2020;72(2):454-469. doi:10.1002/ hep. 31050

28. Zheng J, Zhu L, Iok II, et al. Bone marrow-derived mesenchymal stem cells-secreted exosomal microRNA-192-5p delays inflammatory response in rheumatoid arthritis. Int Immunopharmacol. 2020;78:105985. doi:10.1016/j.intimp.2019.105985

29. Magayr TA, Song X, Streets AJ, et al. Global microRNA profiling in human urinary exosomes reveals novel disease biomarkers and cellular pathways for autosomal dominant polycystic kidney disease. Kidney Int. 2020;98(2):420-435. doi:10.1016/j.kint.2020.02.008
30. Shen Z, Kuang S, Zhang Y, et al. Chitosan hydrogel incorporated with dental pulp stem cell-derived exosomes alleviates periodontitis in mice via a macrophage-dependent mechanism. Bioact Mater. 2020;5(4):1113-1126. doi:10.1016/j.bioactmat.2020.07.002

31. Yuan Q, Chen H, Yang Y, et al. miR-18a promotes Mycobacterial survival in macrophages via inhibiting autophagy by down-regulation of ATM. J Cell Mol Med. 2020;24(2):2004-2012. doi:10.1111/ jcmm.14899

32. Li L, Zuo X, Liu D, et al. The profiles of miRNAs and lncRNAs in peripheral blood neutrophils exosomes of diffuse cutaneous systemic sclerosis. $J$ Dermatol Sci. 2020;98(2):88-97. doi:10.1016/j. jdermsci.2020.02.009

33. Xu Z, Lodge R, Power C, et al. The HIV-1 accessory protein Vpu downregulates peroxisome biogenesis. mBio. 2020;11(2):e03395-19. doi:10.1128/mBio.03395-19.

34. Real JM, Ferreira LRP, Esteves GH, et al. Exosomes from patients with septic shock convey miRNAs related to inflammation and cell cycle regulation: new signaling pathways in sepsis?. Crit Care. 2018;22(1):68. doi:10.1186/s13054-018-2003-3

35. Deng JN, Li YQ, Liu Y, et al. Exosomes derived from plasma of septic patients inhibit apoptosis of $\mathrm{T}$ lymphocytes by down-regulating bad via hsa-miR-7-5p. Biochem Biophys Res Commun. 2019;513 (4):958-966. doi:10.1016/j.bbrc.2019.04.051

36. Li X, He S, Li R, et al. Pseudomonas aeruginosa infection augments inflammation through miR-301b repression of c-Myb-mediated immune activation and infiltration. Nat Microbiol. 2016;1 (10):16132. doi:10.1038/nmicrobiol.2016.132

37. Sang W, Wang Y, Zhang C, et al. MiR-150 impairs inflammatory cytokine production by targeting ARRB-2 after blocking CD28/B7 costimulatory pathway. Immunol Lett. 2016;172:1-10. doi:10.1016/j. imlet.2015.11.001

38. Tan JL, Lau SN, Leaw B, et al. Amnion epithelial cell-derived exosomes restrict lung injury and enhance endogenous lung repair. Stem Cells Transl Med. 2018;7(2):180-196. doi:10.1002/sctm.170185

39. Tang Y, Zhang H, Xu H, et al. Dendritic cells promote treg expansion but not Th17 generation in response to talaromyces marneffei yeast cells. Infect Drug Resist. 2020;13:805-813. doi:10.2147/IDR. S239906
Infection and Drug Resistance

\section{Publish your work in this journal}

Infection and Drug Resistance is an international, peer-reviewed openaccess journal that focuses on the optimal treatment of infection (bacterial, fungal and viral) and the development and institution of preventive strategies to minimize the development and spread of resistance. The journal is specifically concerned with the epidemiology of antibiotic resistance and the mechanisms of resistance development and diffusion in both hospitals and the community. The manuscript management system is completely online and includes a very quick and fair peerreview system, which is all easy to use. Visit http://www.dovepress.com/ testimonials.php to read real quotes from published authors. 